\title{
Rotating Blade Trailing-Edge Noise: Experimental Validation of Analytical Model
}

\author{
Yannick Rozenberg * \\ ONERA \\ 29 avenue de la Division Leclerc, BP72, 92322 Châtillon Cedex, France \\ Michel Roger ${ }^{\dagger}$ \\ Laboratoire de Mécanique des Fluides et d'Acoustique, UMR CNRS 5509 \\ Ecole Centrale de Lyon, 69134 Ecully Cedex, France \\ Stéphane Moreau \\ Groupe d'acoustique de l'Université de Sherbrooke \\ Québec, Canada J1K 2R1
}

\begin{abstract}
The paper is dealing with the experimental validation of an analytical trailing-edge noise model dedicated to low-speed fans operating in free field. The model is intrinsically related to the aerodynamics of the blades and should lead to a useful fast-running tool to be included in a blade design process in an industrial context. The investigations are made on a twobladed low-speed axial fan without shroud, installed inside an anechoic room. The blades are instrumented with two sets of embedded small-size microphones (2.5-mm diameter) and the wall-pressure signals are acquired via a slip ring mounted on the fan axis. The chord-based Reynolds number is about 200000 and the tip Mach number about 0.07 . The data base is completed by far-field measurements made with a single microphone on a moving support. The analytical model is based on a previously published extension of Amiet's trailing-edge noise theory. A blade is split into several strips in the spanwise direction and the model is applied to each strip. For this the input data are interpolated from the measurements performed with the aforementioned sets of microphones. The trailing-edge noise model is more reliable for observer positions within $\pm 30^{\circ}$ from the fan rotation plane.
\end{abstract}

\footnotetext{
*Research Engineer.

${ }^{\dagger}$ Professor. AIAA member.

${ }^{\ddagger}$ Progessor. AIAA member.
} 
Larger differences with the measurements are found when the observer gets closer to the fan axis.

\section{Nomenclature}

\begin{tabular}{|c|c|}
\hline$b_{c}$ & $=$ non-dimensional parameter in Corcos' model \\
\hline$B$ & $=$ fan blade number \\
\hline$c$ & $=$ blade strip chord \\
\hline Co & $=$ sound speed \\
\hline$C_{f}$ & $=$ friction coefficient \\
\hline$k$ & $=$ acoustic wave number \\
\hline$K_{1}$ & $=$ streamwise hydrodynamic wavenumber \\
\hline$L$ & $=$ blade strip span \\
\hline & $=$ spanwise correlation length \\
\hline $\mathcal{L}$ & $=$ aeroacoustic transfer function \\
\hline$M_{t}$ & $=$ tip Mach number \\
\hline$N$ & $=$ fan rotational speed \\
\hline$r$ & $=$ distance between the fan axis and the mid-span network \\
\hline & $=$ distance between the fan axis and the tip network \\
\hline$R$ & $=$ radial distance \\
\hline$R_{0}$ & $=$ distance between observer position and fan center \\
\hline $\overrightarrow{R_{A}}$ & $\begin{array}{l}=\text { position vector of the middle of the trailing-edge segment } \\
\text { in the moving reference frame }\end{array}$ \\
\hline$R_{e}$ & $=$ Reynolds number \\
\hline$S_{0}=\sqrt{x_{1}^{2}+\beta^{2}\left(x_{2}^{2}+x_{3}^{2}\right)}$ & $=$ corrected distance for convection effects \\
\hline$S_{p p}$ & $=$ acoustic pressure power spectral density \\
\hline$S_{p p}^{\Psi}$ & $=$ acoustic pressure power spectral density due to one blade segment \\
\hline$U$ & $=$ tangential velocity \\
\hline$I$ & $=$ convection velocity \\
\hline$\left(x_{1}, x_{2}, x_{3}\right)$ & $=$ moving reference frame \\
\hline$\rightarrow$ & $=$ observer position in the moving reference frame \\
\hline$(X, Y, Z)$ & $=$ fixed reference frame \\
\hline$\vec{Y}$ & $=$ observer position in the fixed reference frame \\
\hline & $=$ airfoil angle of attack \\
\hline$\beta=\sqrt{1-M^{2}}$ & $=$ compressibility factor \\
\hline$\delta^{*}$ & $=$ displacement thickness \\
\hline & $=$ coherence function \\
\hline
\end{tabular}




$\begin{array}{ll}\gamma_{s} & =\text { stagger angle } \\ \eta & =\text { spanwise distance } \\ \phi_{i j} & =\text { cross spectral phase between signals } i \text { and } j \\ \Phi_{p p} & =\text { wall-pressure power spectral density } \\ \tilde{\Phi}_{p p} & =\text { normalized wall-pressure power spectral density } \\ \Theta & =\text { azimuthal observer angle } \\ \rho & =\text { fluid density } \\ \tau_{w} & =\text { wall shear stress } \\ \omega & =\text { radian frequency } \\ \omega_{e} & =\text { emission radian frequency } \\ \Omega & =\text { fan angular velocity } \\ \xi & =\text { streamwise distance }\end{array}$

\section{Introduction}

Considering a simple fan made of a subsonic open rotor, the aerodynamic noise produced by the rotating blades can be divided into tonal noise at the multiples of the blade passing frequency, due to a possible stationary inflow distortion, and broadband noise associated with random fluctuations in the flow. When the ingested flow is highly disturbed, the broadband noise is mainly generated by the scattering of incident turbulence. This mechanism referred to as turbulence-interaction noise has been for instance investigated experimentally for a helicopter rotor and compared with analytical models by Paterson \& Amiet. ${ }^{1}$ In the absence of upstream disturbances, a rotating blade also radiates self noise due to three mechanisms: the generation of vortices at the blade tip, the vortex-shedding possibly occuring due to blunt trailing-edge thickness and the scattering of the boundary-layer turbulence as sound at the trailing edge. The present paper deals with the third mechanism called trailing-edge noise, which corresponds to the minimum noise level radiated by rotating blades without any installation effect or tip clearance, in low-turbulence inflow conditions.

Several analytical trailing-edge noise models applying to airfoils were developed during the seventies, reviewed by Howe. ${ }^{2}$ Later on, Brooks, Pope \& Marcolini ${ }^{3}$ proposed the use of a large database for empirical airfoil self-noise prediction. The trailing-edge noise prediction was found accurate for the high Reynolds number flows at low to moderate angles of attack. A new interest on trailing-edge noise emerged with the recent progress in Computational Fluid Dynamics (CFD) combined with the acoustic analogy. The detailed description of the flow around the airfoil trailing edge of an airfoil has provided the strength of the acoustic sources. $^{4,5}$ Given the source distribution, various numerical or analytical approaches to 
evaluate the trailing-edge noise have been developed. Casper \& Farassat ${ }^{6}$ proposed a timedomain formulation considering a flat plate in a non-uniform flow, based on the fluctuating surface pressure distribution. Zhou \& Joseph ${ }^{7}$ considered airfoils of arbitrary geometries in a uniform mean flow, resorting to a formulation in the frequency domain, and extended the numerical procedure to a rotating blade. ${ }^{8}$

Kim \& George ${ }^{9}$ have also proposed a trailing-edge noise model for open rotors, based on a moving point dipole formula with spanwise loading corrections and assessing the strength of the dipole given by the theory of Amiet. They concluded that trailing-edge noise was important in low inflow turbulence conditions.

The present investigation is similar to the study by Schlinker \& Amiet, ${ }^{10}$ later followed by Moreau et al. ${ }^{11}$ In both references, only the far-field sound was measured and the wallpressure sources were modeled. The difference is that in the present case, a two-bladed fan is instrumented with wall-pressure sensors and a microphone is placed in the far field to measure the radiated noise. The experimental set-up, which can be used to validate trailing-edge noise models, is described in section II. Dedicated sensors have been manufactured and a special care has been taken for their calibration. The wall-pressure statistics, corresponding to the equivalent acoustic sources in the sense of the acoustic analogy applied in various analytical models, is described in section III. The far-field results are presented in section IV. Finally the analytical model is presented in section $\mathrm{V}$, and the trailing edge noise predictions are compared with the acoustic measurements.

\section{Experimental Set-Up}

The present study is aimed at validating isolated-airfoil models for the prediction of low-speed fan trailing-edge noise. The statistics of the aerodynamic pressure induced in the trailing-edge region by the turbulent boundary-layer is most often required for such models. On airfoils, it can be deduced either from dedicated experiments, using for instance remote microphone probes, ${ }^{12-14}$ or from detailed numerical simulations such as Large-Eddy Simulations (LES). ${ }^{4,5,15-17}$ Dealing with a rotating fan blade, LES is still too computationally intensive to model the acoustic sources accurately. One has then to resort to specific detailed measurements to characterize the noise sources. The experimental set-up designed to that goal and and the instrumented fan are described in sections A and B below. Finally, the calibration method is presented in section C.

\section{A. Description of the Test Fan}

The selected fan is a typical industrial ventilation fan (see Figs. 1 \& 3). Its moderately twisted blades are made of plates with a circular-arc mean camber line. The number of 
blades fixed on the hub can be varied between 1 and 12. To avoid additional blade to blade interactions and thus get closer to the behavior of an isolated-blade, only two blades are mounted as described in the next section on a $260 \mathrm{~mm}$-diameter hub. The blade span of $270 \mathrm{~mm}$ leads to a tip radius of $400 \mathrm{~mm}$. For a rotational speed of $600 \mathrm{rpm}$, the blade tip Mach number is $M_{t}=0.07$. The maximum thickness of the blade is $4 \mathrm{~mm}$ and the thickness close to the trailing edge is $3 \mathrm{~mm}$ (at $95 \%$ of the chord length). The chord length slowly varies between $12 \mathrm{~cm}$ and $13.5 \mathrm{~cm}$ from hub to tip. The stagger angle of the blades is $48^{\circ}$ at the hub and $60^{\circ}$ at the tip. More geometrical data are given in Table 1.

\begin{tabular}{lccccccc}
\hline \hline Radius $r(\mathrm{~m})$ & 0.155 & 0.195 & 0.235 & 0.275 & 0.315 & 0.355 & 0.395 \\
\hline Chord length $(\mathrm{m})$ & 0.12 & 0.12 & 0.125 & 0.13 & 0.13 & 0.135 & 0.135 \\
\hline Stagger angle $\left(^{\circ}\right)$ & 47.8 & 49.7 & 51.9 & 53.8 & 55.6 & 57.7 & 59 \\
\hline \hline
\end{tabular}

Table 1: Geometrical data of the blade.

To focus on the self-noise mechanism only, great care has been taken to reduce other possibly contributiong mechanisms. Therefore, the fan is placed in a quiet environment in the middle of the ECL-LMFA (Laboratoire de Mécanique des Fluides et Acoustique de l'Ecole Centrale de Lyon) anechoic chamber far from any additional solid surface close to the blades, as shown in Fig. 1. The motor and the support struts are assumed to have no significant effect. Nevertheless, hot-wire measurements have been carried out in a plane upstream of the fan to check that the incoming flow is not turbulent enough to induce turbulence-interaction noise. Some tuft flow visualizations (see Fig.20 in Rozenberg et al. ${ }^{18}$ ) have also shown that at the present stagger angle, the tip vortex detaches from the blade tip without interacting with the trailing edge. Furthermore the limited number of blades ensures that the tip vortex from one blade is convected by the mean flow without interaction with the following blade. Finally, trailing-edge noise and vortex-shedding noise remain as the only possible contributors. However, the stagger angle of the blade leads to a relatively high local angle of attack and consequently vortex-shedding noise (also called blunt trailing-edge noise) is unlikely to occur, as observed by Hutcheson \& Brooks. ${ }^{19}$ It is then considered that the measured broadband noise in the present experiment is essentially trailing-edge noise.

The anechoic room is designed to ensure nearly-zero reflection above the low-frequency limit of $100 \mathrm{~Hz}$. A half-an-inch Brüel and Kjaer 4181 free-field microphone is mounted on a rotating arm, at a distance $R_{0}=1.7 \mathrm{~m}$ away from the center of the fan (cf. Fig. 1). This distance leads to a value of $k R_{0}=3$ for the lowest frequency of interest (100 Hz), compatible with the condition of acoustic far-field at higher frequencies. This condition is implicitly assumed in the models. The microphone sensitivity has been previously calibrated using a Brüel and Kjaer pistonphone at $1 \mathrm{kHz}$. The set-up allows to investigate the noise directivity 
within the range $-130^{\circ}<\Theta<130^{\circ}$, where $\Theta$ is the angle between the axis of rotation and the microphone. The far-field spectra could be reproduced with an accuracy below $1 \mathrm{~dB}$. Reliable and repeatable measurements are achieved for $30^{\circ}<|\Theta|<130^{\circ}$. For a microphone angle less than $30^{\circ}$, the microphone is in the fan exhaust jet and the acoustic measurements are spoiled by pseudo-sound.

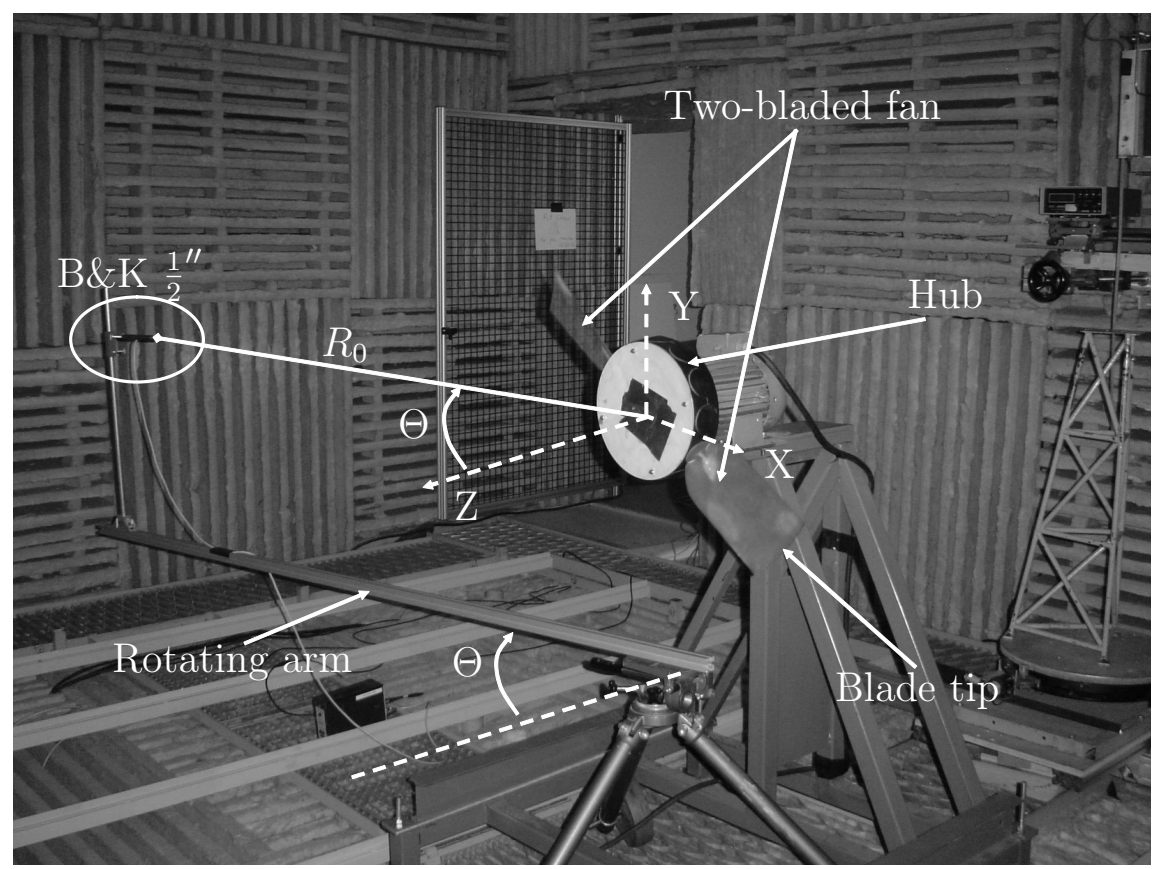

Figure 1: Experimental set-up for far-field studies: the slip ring is removed and the sensors embedded in the blades are not connected.

\section{B. Fan Blade Instrumentation}

Recent improvements in electronics make the mounting of small-size microphones on moving fan blades possible. Here the microphones selected for the unsteady wall-pressure measurements are FG-3329-P07 manufactured by Knowles Acoustics. They have a diameter of $2.5 \mathrm{~mm}$ and a length of $2.5 \mathrm{~mm}$, small enough to be inserted inside the blade parallel to the surface but not enough to be normally flush mounted. The connections also prevent such a mounting perpendicular to the surface.

The microphones and their connection cables are maintained in aluminum tubes along grooves just beneath the surface and communicate with the surrounding air through a $0.6 \mathrm{~mm}$-diameter lateral pinhole which is the actual measuring point. This mounting features a small cavity between the active section of the microphone and the hole, acting as a Helmholtz resonator at high frequencies, as shown on the cross-section view of Fig. 2. The pinholes are small enough to resolve most scales of the turbulent boundary layer as shown 
in section $\mathrm{C}$. The complete system, including the microphone and the perforated cavity will be called a sensor later on in the paper. A 14-channel slip ring on the fan axis allows transmitting the signals from the rotating sensors to the processing unit. Its effect on the signal is found to be insignificant in the frequency range of interest. All the measurements are collected with a 16-channel HP3565 Paragon acquisition system. The processing is made between 0 and $12800 \mathrm{~Hz}$ with a constant bandwidth of $4 \mathrm{~Hz}$ and provides the power spectral density (PSD) of the wall-pressure fluctuations in $\mathrm{dB} / \mathrm{Hz}$ with a reference pressure of $2.10^{-5} \mathrm{~Pa}$. The number of averages $\left(N_{i}=400\right)$ has been chosen to provide an experimental uncertainty of $0.2 \mathrm{~dB}$ on the wall-pressure spectra. Reliable and repeatable measurements are achieved for all sensors over a range $100 \mathrm{~Hz}-10 \mathrm{kHz}$, except for sensors 6 , E and F, which were damaged during their insertion inside the blade. So the wall pressure spectra given by these three sensors are not used in the present study.

The sensors are arranged in two networks of 6 for the sake of determining the statistics of the aerodynamic pressure, as shown in Fig. 3. The mid-span network is placed at a distance $r_{1}=270 \mathrm{~mm}$ from the fan axis on one blade and the tip network at $r_{2}=357 \mathrm{~mm}$ on the other one.

The sets of sensors (1 to 4 ) and (A to D) distributed along the span provide the spanwise coherence length, whereas streamwise aligned sensors with appropriate post-processing provide information on the convection speed of the turbulent eddies. Special care was taken to select the distance of the U-shaped networks from the trailing edge. On the one hand, the measurement of the incident field must not be affected by the scattering process occurring at the trailing edge. On the other hand, it must be representative of the turbulence properties past the trailing edge. According to Ffowcs-Williams \& Hall ${ }^{20}$ and Brooks \& Hodgson, ${ }^{21}$ the distance must be more than the hydrodynamic wavelength $d \approx \lambda_{h}=U / f$. It can be larger if the wall-pressure fluctuations are statistically homogeneous in the aft part of the blades. Here, the closest sensors are $10 \mathrm{~mm}$ away from the trailing edge, which corresponds to a typical hydrodynamic wavelength for a frequency of $570 \mathrm{~Hz}$ at a radius $R=270 \mathrm{~mm}$, for a rotating speed of $200 \mathrm{rpm}$. At the blade tip, it corresponds to $740 \mathrm{~Hz}$. So, it can be considered that the measured incident surface pressure is not contaminated by the scattered pressure field above these frequencies.

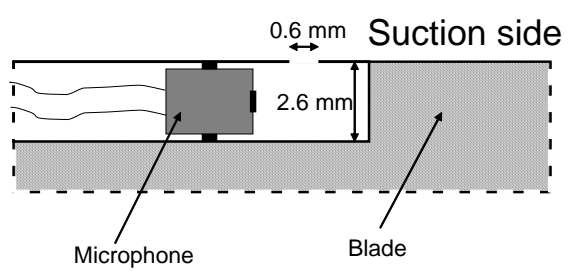

Figure 2: Description of the sensor geometry. Cross-section view. 

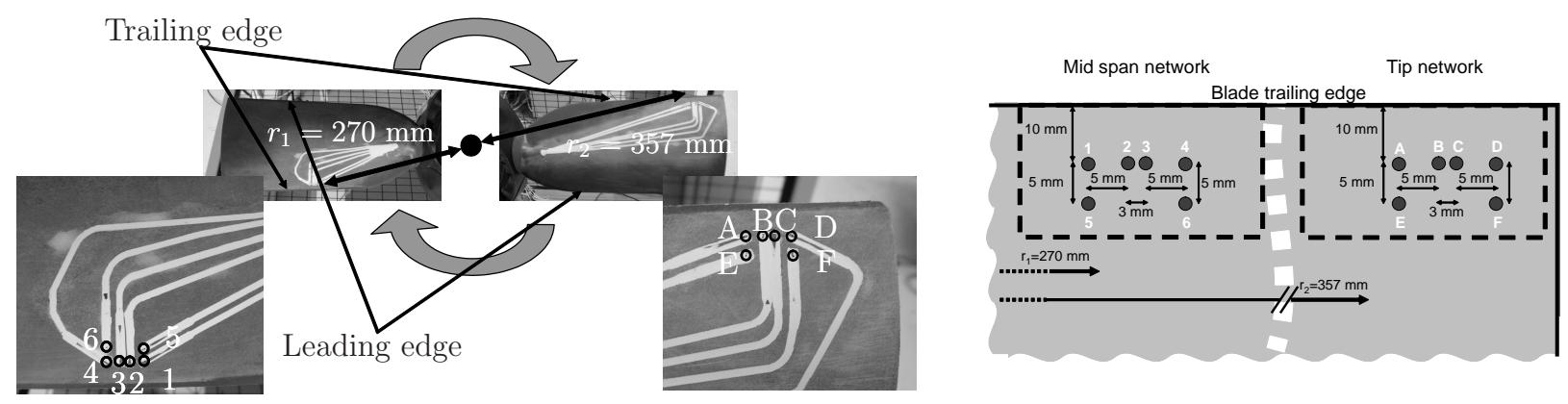

Figure 3: Fan geometry and sensor set-up in each blade

\section{Sensor Calibration}

First, the sensors are calibrated using a pistonphone generating broadband noise. A reference microphone (Brüel \& Kjaer 1/4") provides the transfer function for each sensor. To check the ability of the sensor to resolve the turbulent boundary layer scales, a comparison is provided for a zero-pressure-gradient wall-pressure spectrum. The results obtained with the sensor are compared with the spectrum given by a Brüel \& Kjaer 1/4" microphone. The attenuation at high frequencies due to the spatial averaging of small hydrodynamic wavelengths is taken into account for the $1 / 4$ " microphone, using the correction proposed by $\operatorname{Corcos}^{22}$ with an equivalent radius ${ }^{23} r_{e q}=0.62 r$ and a convection velocity $U_{c}=0.7 U, U$ being the flow velocity in the middle of the wind tunnel section and $r$ the actual radius of the microphone. The wall-pressure spectra measured with the sensor and the reference microphone perfectly match between $300 \mathrm{~Hz}$ and $3000 \mathrm{~Hz}$. Between $3000 \mathrm{~Hz}$ and $6000 \mathrm{~Hz}$ a difference of approximately $3 \mathrm{~dB}$ is observed. It corresponds to the Helmholtz resonance frequency range of the cavity. At higher frequencies, the agreement is acceptable (discrepancies less than $1 \mathrm{~dB}$ ).

Anyway, the sensor technology leads to specific calibration issues. They have been discussed by Rozenberg et al. ${ }^{18}$ who demonstrated by additional tests that sensors mounted on the blades have a non linear behavior when the fan rotates at $600 \mathrm{rpm}$. The time signals, not shown here, exhibited a clear saturation. Facing this difficulty, an alternative calibration procedure has been defined as follows. The idea is to decrease the rotational speed down to a value for which the linear behavior of the sensor is obviously recovered becaue the mesaured fluctuations are weaker, and the transfer function is valid. The measured wall-pressure spectrum is then made non-dimensional using mixed variables as proposed by Keith et al. ${ }^{24}$ namely the tangential velocity $U$, the displacement thickness $\delta^{*}$ and the wall shear stress $\tau_{w}$. Finally, it is rescaled to a higher speed for which the measurements are not reliable. The displacement thickness and wall shear stress are deduced from the $1 / 7^{\text {th }}$-power velocity distribution law, which is the most common power law for the turbulent boundary layer without any pressure gradient. It leads to the classical formulations where $R e$ is the 


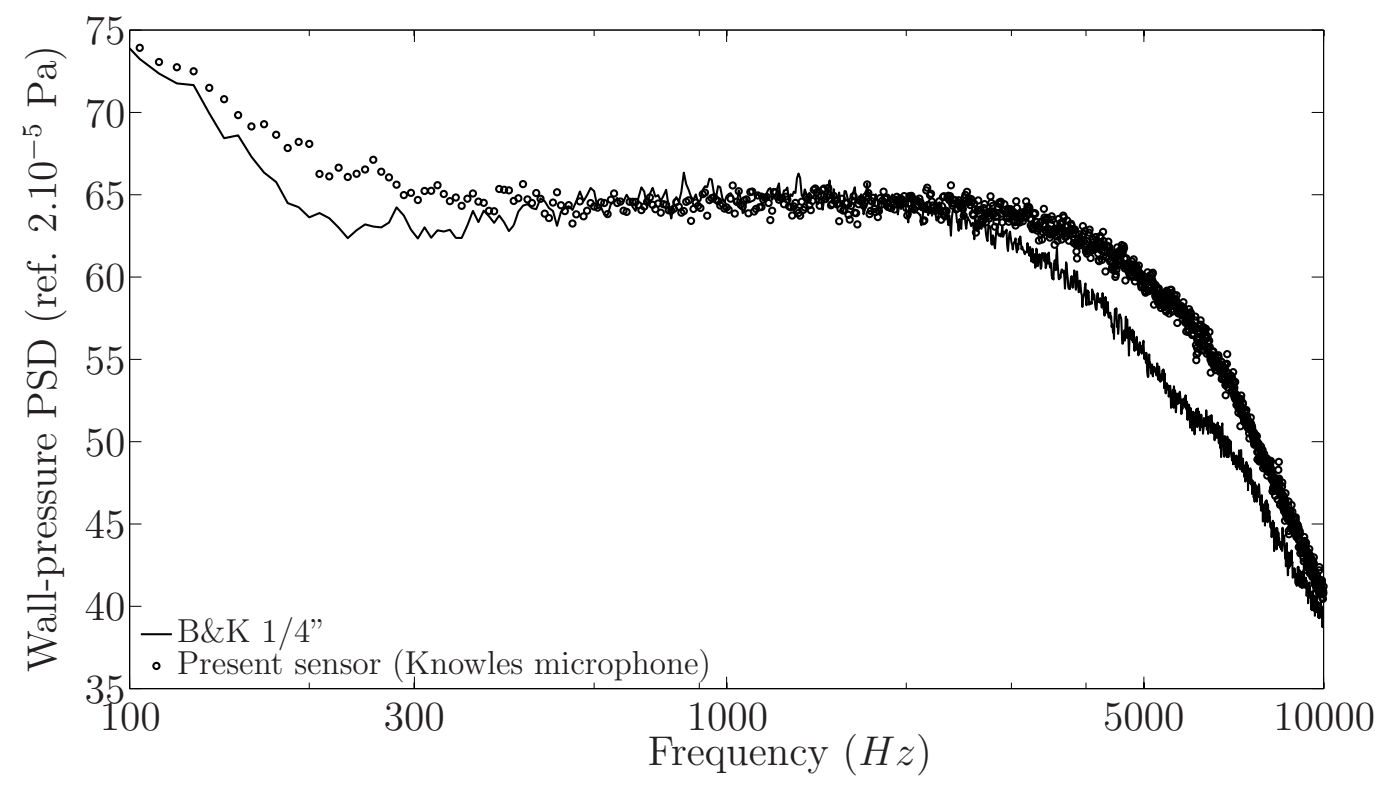

Figure 4: Wall-pressure spectrum beneath a zero-pressure-gradient turbulent boundary layer measured by a B\&K 1/4" microphone (-) and a Knowles sensor (o). Mean velocity: $U=20$ $\mathrm{m} / \mathrm{s}$.

Reynolds number based on the mean velocity and the chord: ${ }^{25}$

$$
\frac{\delta^{*}}{c}=\frac{0.0477}{R_{e}^{1 / 5}} \quad, \quad C_{f}=\frac{0.0594}{R_{e}^{1 / 5}} \quad \text { with } \quad \tau_{w}=\frac{1}{2} \rho U^{2} C_{f}
$$

The non-dimensional spectrum reads:

$$
\widetilde{\Phi}_{p p}=\frac{\Phi_{p p} U}{\delta^{*} \tau_{w}^{2}}
$$

Plotting the direct measurements of $\widetilde{\Phi}_{p p}$ as a function of the Strouhal number $S t$ based on the displacement thickness and the tangential velocity $U=\Omega R$ of the fan blades for different rotational speeds should make the curves collapse, assuming the self-similarity of the flow. This is carried out for 6 rotational speeds between $200 \mathrm{rpm}$ and $594 \mathrm{rpm}$. The inspection of the time signals confirmed that the saturation occurs clearly for the three highest rotational speeds. As a consequence, the normalized spectra do not collapse for these configurations, as observed in Fig. 5 for sensor 1. For the lower velocities, the discrepancies are reduced and the resonance due to the cavity is attenuated and even avoided at $200 \mathrm{rpm}$. This is why the data collected at a rotational speed of $200 \mathrm{rpm}$ are best suited for the calibration procedure. Then they are normalized and extrapolated to $600 \mathrm{rpm}$ using formula (1). The results are presented in the following section. It must be noted that the procedure is based on the 
assumption of self-similar flows at different speeds. This has been confirmed by inspection of the measured sound spectra, which follow a clear similarity scaling law.

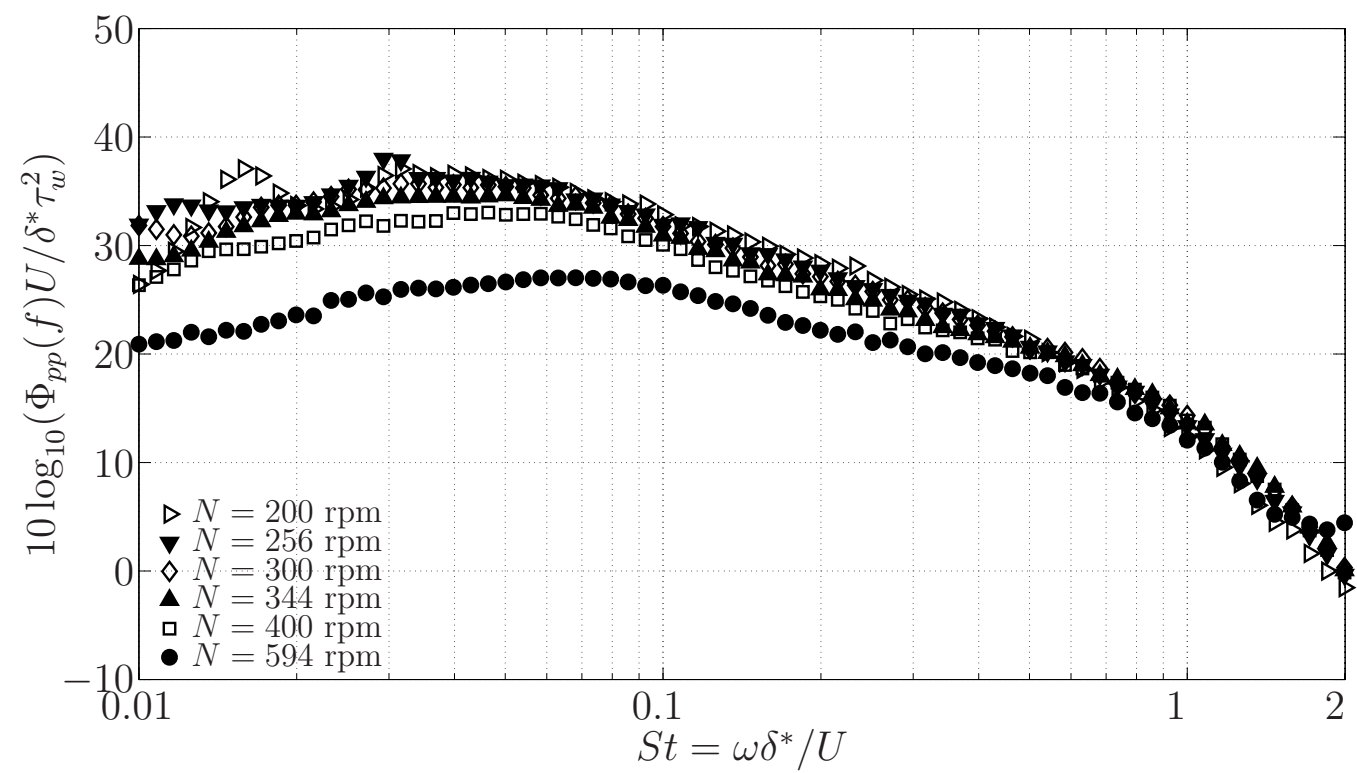

Figure 5: Wall-pressure spectra from the sensor 1 on the rotating blade normalized using eq. (1).

\section{Measured Wall-Pressure Statistics}

The embedded sensors are used to collect the statistical features of the wall-pressure fluctuations. Wall-pressure spectra are first studied here to check the turbulence homogeneity in both instrumented blade areas. Then the convection velocity of the turbulent eddies is deduced from phase measurements between streamwise-aligned sensors. Finally, a length scale is defined from the coherence between spanwise sensors.

\section{A. Wall-Pressure Spectra}

The wall-pressure spectra from the mid-span and the blade-tip sensor networks, after calibration according to the procedure described in section II.C, are shown in Figs. 6 and 7. The spectra measured by the mid-span network and plotted in Fig. 6 collapse, confirming the expected turbulence homogeneity. They exhibit different slopes in three frequency ranges: a first one $\left(f^{-1}\right)$ attributed to large eddies, a second one $\left(f^{-7 / 3}\right)$ to middle-size eddies and a third one $\left(f^{-5}\right)$ to smaller eddies. Despite the lack of local aerodynamic data, the present slopes can be compared with those observed around airfoils by previous investigators. The $(-1)$ slope is observed in the overlap region, corresponding to Bradshaw's inactive motion. ${ }^{26}$ 
Its frequency range depends on the Reynolds number. The higher this number is, the wider the frequency domain of the overlap region. In this region, the wall-pressure spectra can be normalized by both the inner and the outer boundary-layer variables. These results found theoretically by Bradshaw ${ }^{26}$ have also been observed experimentally by McGrath \& Simpson. ${ }^{27}$ The second slope $(-7 / 3)$ has been deduced by Monin \& Yaglom ${ }^{28}$ from a theoretical study on the locally isotropic turbulence. Finally, the $(-5)$ slope is considered by Blake as typical of the inner-layer turbulence, ${ }^{29}$ i.e. very close to the wall. It has been experimentally observed by Gravante et al. ${ }^{30}$ and McGrath \& Simpson ${ }^{27}$ and in a direct numerical simulation of a pipe-flow with adverse pressure gradient by Na \& Moin. ${ }^{31}$ Thus, the present mid-span wall-pressure spectra are quite similar to several canonical flows.

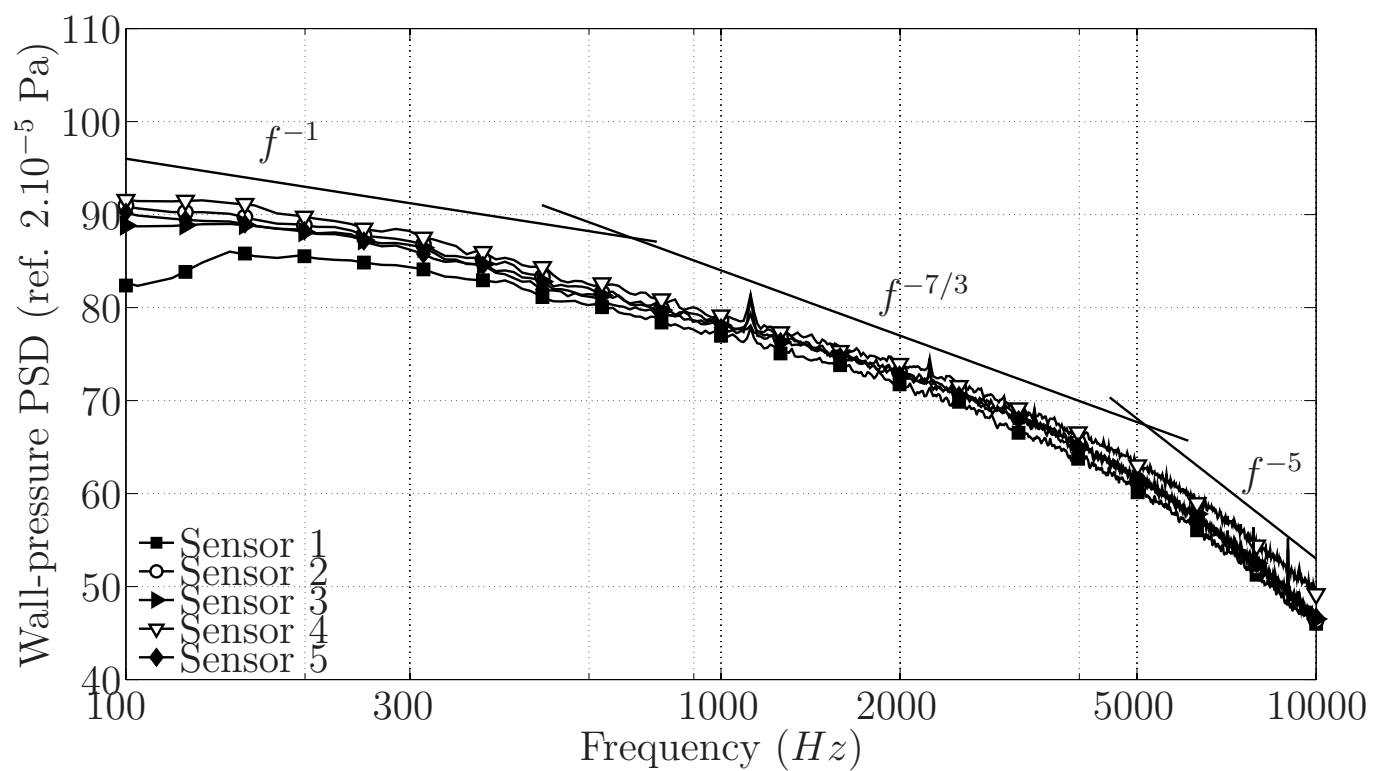

Figure 6: Wall-pressure PSD transposed to $N=600 \mathrm{rpm}$ from measurements at $N=$ 200 rpm. Mid-span network.

In contrast, a significant local non-homogeneity of the pressure field near the blade tip is suggested by the wall-pressure spectra measured in that region and prevents them from convincingly collapsing, as shown in Fig. 7. This makes the definition of characteristics slopes from the spectra questionable. The blade-tip spectra are very different from the mid-span spectra both in terms of spectral shape and level. In this configuration, the inertial sublayer characterized by the $\left(f^{-5}\right)$ slope has almost disappeared. Goody \& Simpson ${ }^{32}$ in their study of a wing-body junction flow observed wall-pressure spectra with higher levels when the flow is fully three-dimensional, which seems to be the case in the blade tip. Therefore, the nonhomogeneity of the wall-pressure spectra suggests the presence of a three-dimensional flow, possibly due to the induction of the expected detached vortex formed near the blade tip. Nevertheless the scatter in the data does not exceed 4 to $6 \mathrm{~dB}$ depending on the frequency, 
and remains compatible with a use as input data for noise evaluation, provided that it is considered as responsible for a margin of uncertainty.

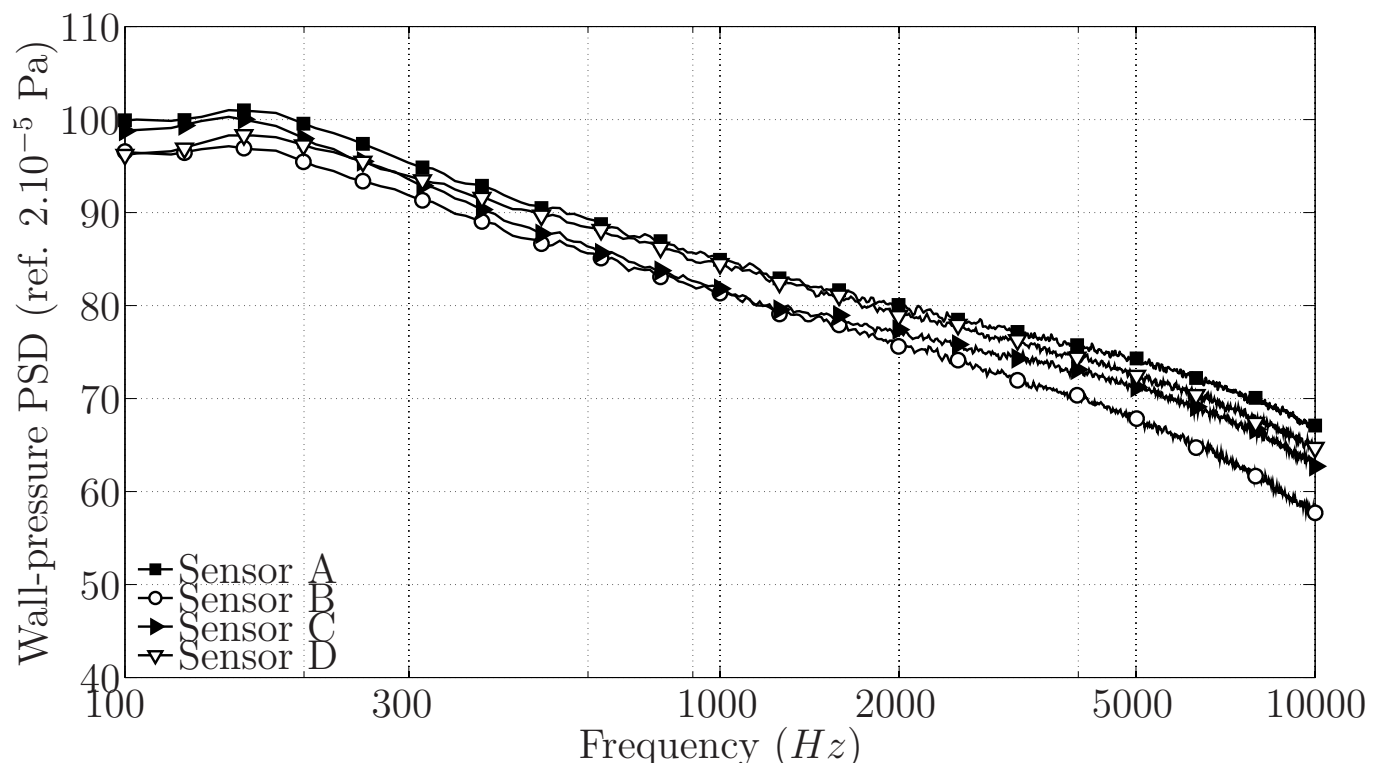

Figure 7: Wall-pressure PSD transposed to $N=600 \mathrm{rpm}$ from measurements at $N=$ 200 rpm. Blade-tip network.

\section{B. Convection Velocity}

Since the lifespan of an eddy is proportional to its scale, ${ }^{21}$ only the larger eddies remain coherent between two widely separated points. Moreover, larger eddies are farther from the surface and are convected at a higher velocity. So the convection velocity is an increasing function of the distance $\xi$ between sensors, as pointed out by Brooks \& Hodgson. ${ }^{21}$ Because the number of embedded sensors is limited, only a single streamwise separation distance $(\xi=5 \mathrm{~mm})$ is available to evaluate this quantity. Corcos ${ }^{33}$ defined an average convection velocity $U_{c}$ based on the cross-spectral phase $\phi_{i j}$ between the sensors $i$ and $j$ separated by a distance $\xi$ as:

$$
\phi_{i j}=\frac{\omega \xi}{U_{c}(\omega, \xi)}
$$

The cross-spectral phase is plotted in Fig. 8 for each configuration. On each set, two separate cross spectra are post-processed giving more confidence in the results summarized in Table 2. The convection velocity deduced from the tip network is found higher than the tangential velocity $(U=22.4 \mathrm{~m} / \mathrm{s})$, highlighting the three-dimensional effect of the vortical structures near the blade tip. 


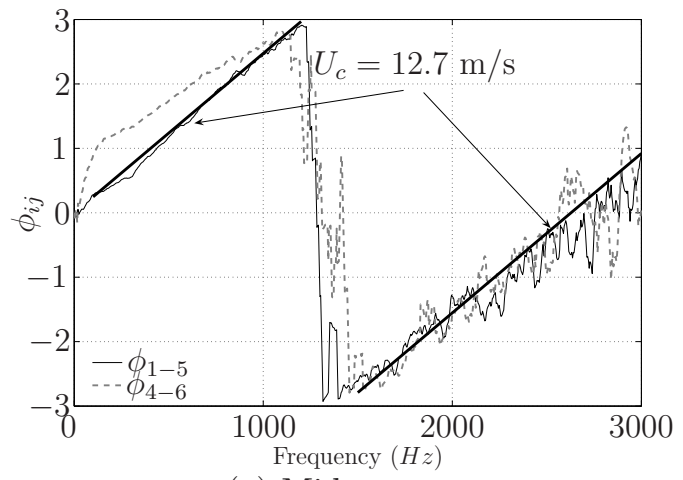

(a) Mid-span set



(b) Blade-tip set

Figure 8: $\phi_{i j}$ : cross spectral phase between sensor $i$ and sensor $j$. Black thick lines correspond to Eq. (2).

\begin{tabular}{cccc}
\hline \hline & $U_{c}(\mathrm{~m} / \mathrm{s})$ & $U_{c} / U$ & $b_{c}$ \\
\hline mid-span set & 12.7 & 0.75 & 1.56 \\
\hline blade tip set & 24 & 1.07 & 0.9 \\
\hline \hline
\end{tabular}

Table 2: Convection velocity and Corcos' model constant in the present experiment.

\begin{tabular}{lcccc} 
& $U_{0}$ & $U_{c}$ & $U_{c} / U_{0}$ & $b_{c}$ \\
\hline \hline $\begin{array}{l}\text { Flat plate }^{34}\left(\alpha_{g}=5^{\circ}\right) \\
\text { LMFA small wind-tunnel }\end{array}$ & $40 \mathrm{~m} / \mathrm{s}$ & $32 \mathrm{~m} / \mathrm{s}$ & 0.8 & 2 \\
\hline $\begin{array}{l}\text { CD airfoil } \\
\text { LMFA large wind-tunnel }\left(\alpha_{g}=15^{\circ}\right)\end{array}$ & $16 \mathrm{~m} / \mathrm{s}$ & $12 \mathrm{~m} / \mathrm{s}$ & 0.75 & 1.2 \\
\hline $\begin{array}{l}\text { CD airfoil } \\
\text { LMFA large wind-tunnel }\left(\alpha_{g}=8^{\circ}\right)\end{array}$ & $16 \mathrm{~m} / \mathrm{s}$ & $11.2 \mathrm{~m} / \mathrm{s}$ & 0.7 & 1.5 \\
\hline $\begin{array}{l}\text { CD airfoil } \\
\text { LMFA small wind-tunnel }\left(\alpha_{g}=13^{\circ}\right)\end{array}$ & $16 \mathrm{~m} / \mathrm{s}$ & $9.6 \mathrm{~m} / \mathrm{s}$ & 0.6 & 1.5 \\
\hline $\begin{array}{l}\text { V2 airfoil } \\
\text { LMFA small wind-tunnel }\left(\alpha_{g}=20^{\circ}\right)\end{array}$ & $16 \mathrm{~m} / \mathrm{s}$ & $10.4 \mathrm{~m} / \mathrm{s}$ & 0.65 & 1.4 \\
\hline
\end{tabular}

Table 3: Convection velocity and Corcos' model constant from typical airfoil experiments. ${ }^{12,14,34,35}$ 


\section{Spanwise Correlation Length Scale}

The spanwise correlation length is defined as:

$$
l_{y}(\omega)=\int_{0}^{\infty} \sqrt{\gamma^{2}(\omega, \xi=0, \eta)} d \eta
$$

where $\gamma^{2}$ is the coherence function, an example of which is plotted in Fig. 9. Between $300 \mathrm{~Hz}$ and $3000 \mathrm{~Hz}$, the coherence decreases with both frequency and sensor separation. This agrees with Corcos' model, postulating an exponential decrease with both parameters:

$$
\gamma^{2}(\omega, \xi=0, \eta)=\mathrm{e}^{-\frac{2 \omega}{b_{c} U_{c}} \eta} .
$$

Knowing $U_{c}$ and plotting the logarithm of the coherence as a function of the frequency, a linear regression of the experimental data provides the coefficient $b_{c}$. The values determined this way are compared in Table 2 with the reference value for a turbulent boundary layer over a flat plate obtained by $\operatorname{Corcos}^{33}\left(b_{c}=1.4\right)$ and the data collected on stationary airfoils at the LMFA-ECL facilities (see Table 3). As the blade profile is basically a plate with a circular mean line, the values obtained at the mid-span for $b_{c}$ and the ratio $U_{c} / U$ are similar to the results obtained on the flat plate and the CD airfoil, which is also characterized by a circular arc mean line. In Fig. 9, a poor agreement is observed between the measured coherence on the mid-span set and Corcos' model. The predictions are in a better agreement with the coherence determined from the blade-tip set. The maximum error made on Corcos' coefficient $b_{c}$ is $30 \%$. Since the coherence length scale defined by the model can be written $l_{y}=b_{c} U_{c} / \omega$, the maximum error on $l_{y}$ is also $30 \%$. This error will be taken into account in the section V.B to evaluate the numerical inaccuracy of the prediction induced by the data uncertainties.

At low frequencies, the decrease observed in the different plots is attributed to the finite size of the turbulent eddies. Indeed, the spanwise correlation length is characteristic of the size of turbulent eddies, restricted by the boundary layer thickness.

At high frequencies, the measured coherence is very low and is no longer decreasing with frequency. But the measurement is not reliable below a threshold imposed by data processing constraints. The larger the number of averages is, the lower the threshold is. Due to this limitation, Corcos' assumption of continuously decreasing coherence with frequency is more relevant, once confirmed by the measurements at lower frequencies. So, an adhoc model for the spanwise coherence length is used here. At low frequencies (between $100 \mathrm{~Hz}$ and $800 \mathrm{~Hz}$ ), the length scale is defined by the experimental results using Eq. (3). Between $1200 \mathrm{~Hz}$ and $10 \mathrm{kHz}$, the coherence function is below the confidence limit roughly defined 
by $1 / \sqrt{N_{i}}$, where $N_{i}=400$ is the number of averages. Corcos' model is retained in this range. Finally, between $800 \mathrm{~Hz}$ and $1200 \mathrm{~Hz}$, a linear interpolation is defined between the low-frequency model and the high-frequency model.

The spanwise coherence length detemined this way is presented in Fig. 10. Flow visualizations using tuft have shown evidence of large structures at the blade tip. ${ }^{18}$ These structures are supposed to have an influence on the blade-tip set of sensors. As a result, the maximum of the coherence length is obtained at a lower frequency in this region.

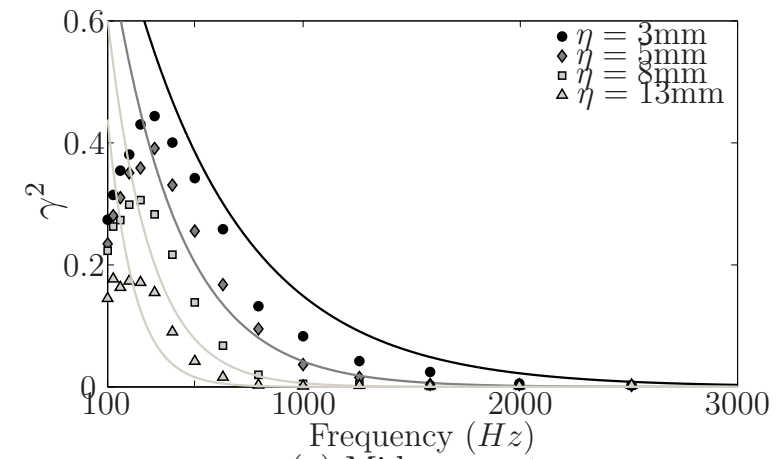

(a) Mid-span set

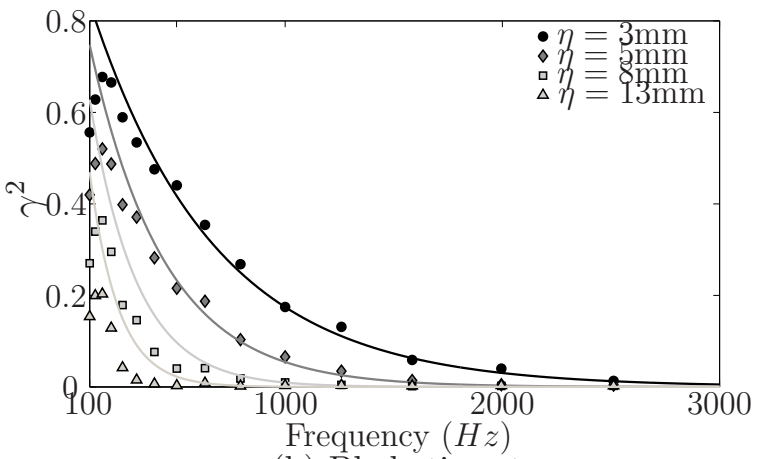

(b) Blade-tip set

Figure 9: Coherence function $\gamma^{2}$. Experimental results (symbols) and Corcos' model (lines).

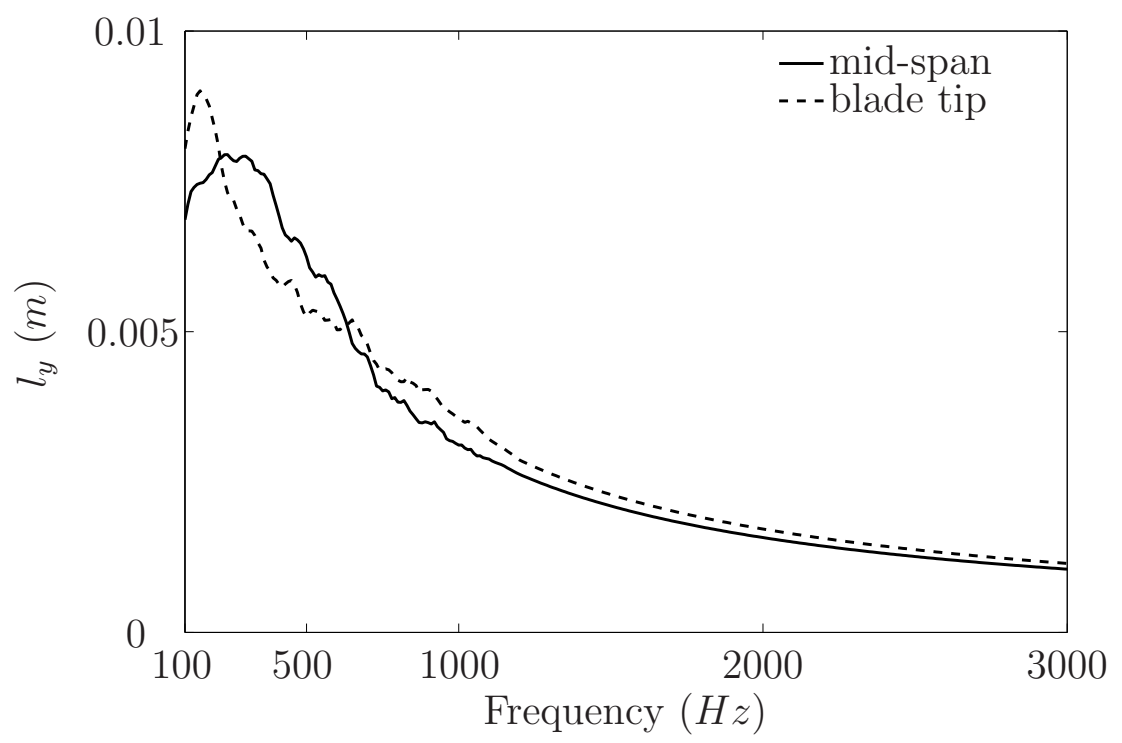

Figure 10: Spanwise coherence length obtained by adhoc model: experimental results at low frequencies and Corcos' model at high frequencies. 


\section{Far-Field Results}

The experimental set-up described in section II and in Fig. 1 allows studying the acoustic far-field in reliable anechoic conditions. In the present section the scaling of the far-field pressure with the rotational speed is addressed first. Then an investigation of the directivity is described.

\section{A. Scaling with Rotational Speed}

According to the acoustic analogy, the overall sound intensity radiated by a dipole is proportional to the $6^{\text {th }}$ power of a characteristic velocity. Hence, a scaling law of the pressure PSD normalized by $U^{5}$ as a function of the Strouhal number $S t=f c / U$ based on the chord length should be found. This is based on the assumptions of self-similar flows and compact acoustic sources. Recent experimental results on airfoils ${ }^{12,36}$ suggest a scaling with $U^{n}$ where the exponent $n$ varies between 4 and 5 . Brooks \& Hodgson ${ }^{21}$ and Schlinker \& Amiet ${ }^{10}$ obtained $n=5$. Deviations from this exponent emphasize the chordwise non-compactness of the airfoil at the measured frequencies. The same trend and values for $n$ have been observed by Stephens \& Morris ${ }^{37}$ on a ducted rotor for different operating points. For the present fan, the far-field acoustic spectra in the rotational plane were measured for 7 rotational speeds between $400 \mathrm{rpm}$ and $1000 \mathrm{rpm}$. The normalization by $U^{5}$ provides a good collapse as can be seen in Fig. 11. This result provides a first a posteriori justification of the assumptions made for the sensor calibration in section II.C, namely the self-similarity of the flow.

It should be stressed that the trailing-edge noise model $^{38}$ assumes relatively high frequencies and holds in the limit of low frequencies down to $k c \approx 1 / 4$, thus typically $100 \mathrm{~Hz}$ in the present application. Despite the non-compactness, the $5^{\text {th }}$-power law remains acceptable also at higher frequencies. This can be explained by the progressive concentration of the induced lift fluctuations closer to the trailing edge as the frequency increases, as stated for a flat plate by Amiet's model. ${ }^{39}$ It must also be noted that the low frequencies in the present experiment correspond to a range in which the leading-edge back-scattering correction shown below in section V.A is needed. ${ }^{38}$

\section{B. Directivity Measurements}

The only difference between a first observer at a position $\left(R_{0} ; \Theta\right)$ and a second one at $\left(R_{0} ;-\Theta\right)$ results from the rotation of the blades. But considering an averaged sound pressure level for random sources with homogeneous properties along their path, the noise perceived by both observers must be the same. This symmetry is highlighted in Fig. 12. The background noise is also plotted. It is defined as the noise measured with the electric motor turned on and the blades removed. The fan broadband noise clearly exceeds the background noise by 


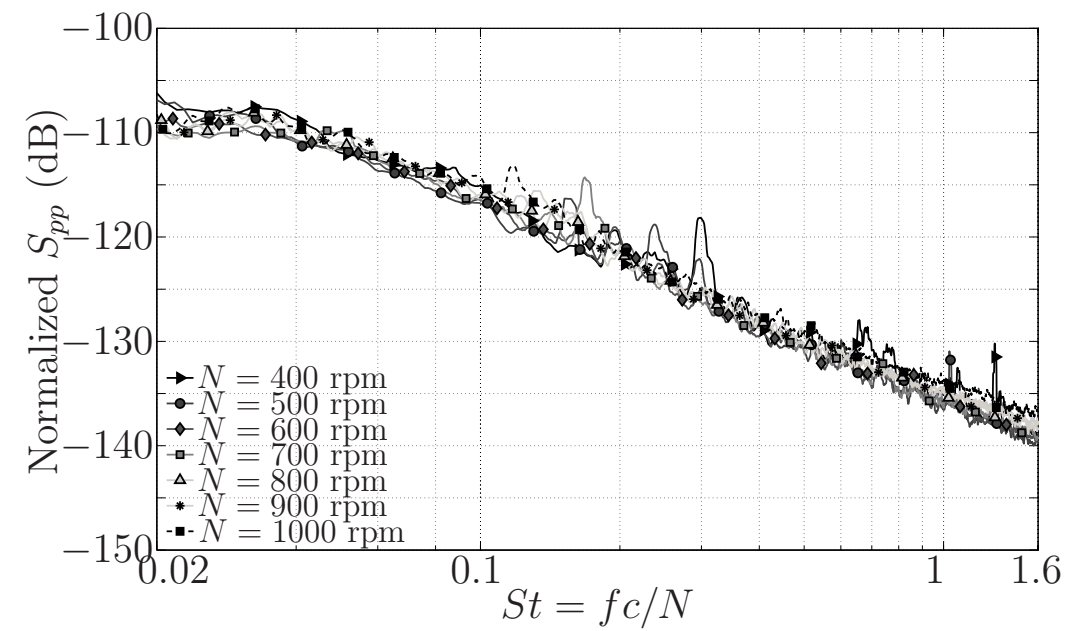

Figure 11: Effect of the rotational speed on the sound radiated in the rotation plane.

$10 \mathrm{~dB}$ over almost the whole frequency range of interest, typicallty between $100 \mathrm{~Hz}$ and 10 $\mathrm{kHz}$. The data can be used unambiguously as a basis for validation with no need for any subtraction procedure, and preserving uncertainties below $1 \mathrm{~dB}$. Only some limited tonal noise components coming from the electric motor contaminate the fan noise measurements, but they are not prejudicial to the present study.

\section{Trailing-Edge Noise Model for a Rotating Blade}

\section{A. Model Background}

In 1976, Amiet published an isolated-airfoil trailing-edge noise model ${ }^{39}$ based on a theoretical background named Schwarzchild's technique, previously proposed for predicting the broadband noise of an airfoil in an incident turbulent flow. ${ }^{40}$ More recently Roger \& Moreau ${ }^{38}$ extended the trailing-edge noise formulation to account for the effects of a finite chord length, by including a leading-edge back-scattering correction. Schwarzschild's technique provides the chordwise unsteady lift distribution which acts as the acoustic sources. The far-field noise is then evaluated by applying the classical formula for the radiation of a dipole in a uniform flow, via a statistical analysis. The final result, namely the far-field noise PSD produced by the scattering of the turbulent boundary layer past the trailing edge, is expressed below in the reference frame centered at the trailing edge of the airfoil at mid-span. $x_{1}$ is aligned with the inflow velocity, $x_{3}$ is perpendicular to both the trailing edge and $x_{1}$, with $x_{3}=0^{+}$on the suction side. Finally, $x_{2}$ is aligned with the trailing edge to obtain a direct system. The turbulence is supposed frozen in a small region near the trailing edge, so that the streamwise wavenumber of its pressure signature at the wall is equal to $K_{1}=\omega / U_{c}$. Assuming a large 


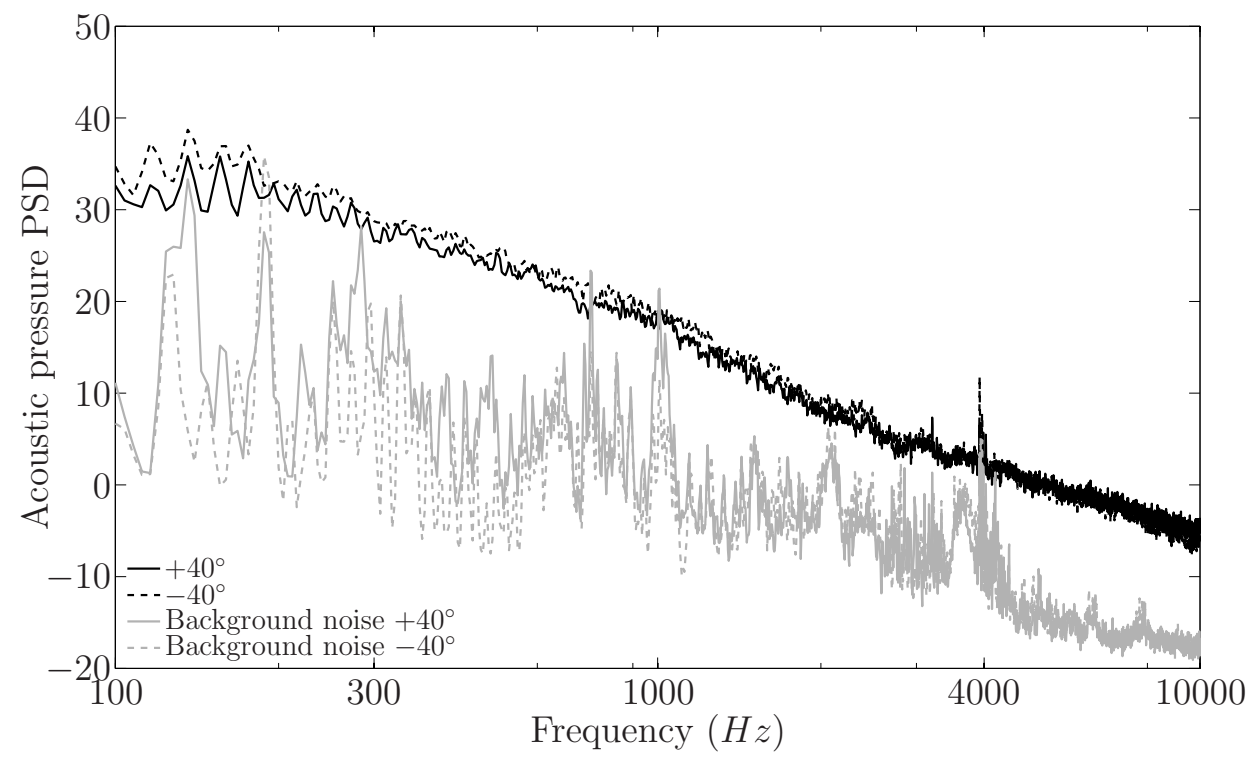

(a) $\Theta= \pm 40^{\circ}$

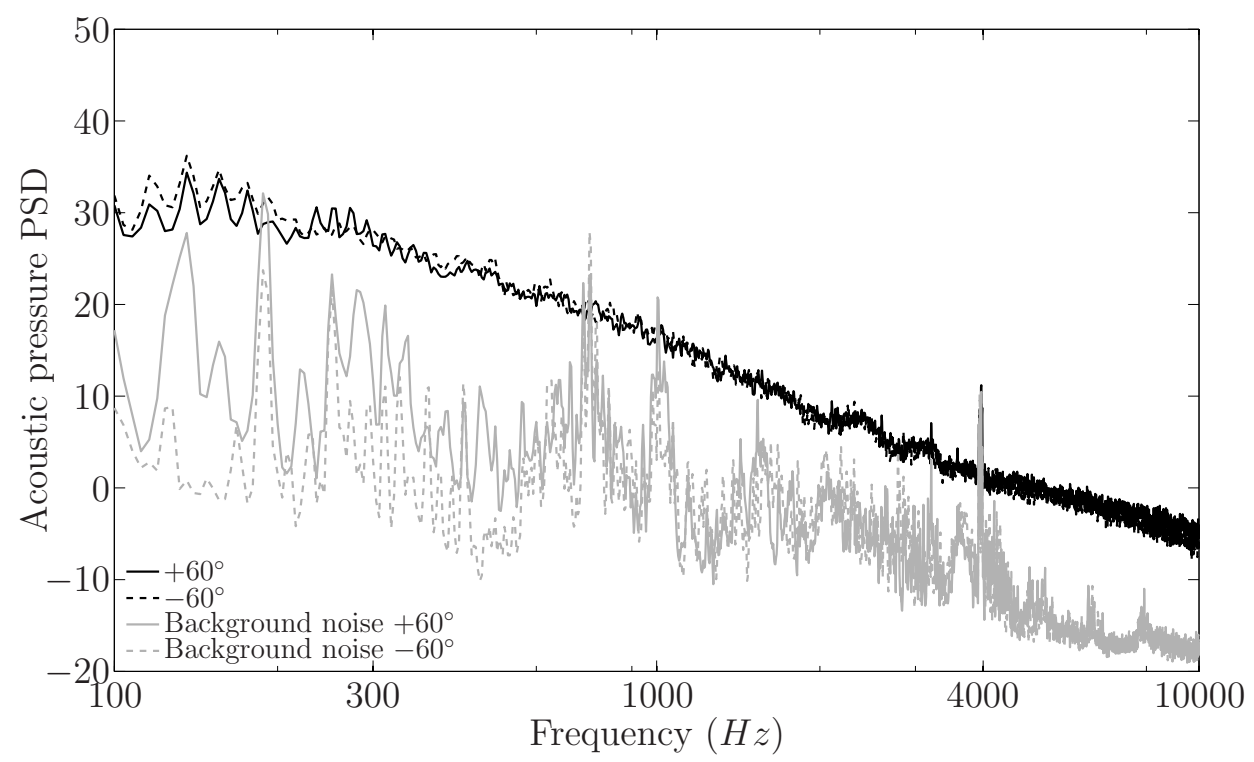

(b) $\Theta= \pm 60^{\circ}$

Figure 12: Far field acoustic pressure PSD (dB/Hz ref. $2.10^{-5} \mathrm{~Pa}$ ). Results at $R=1.7 \mathrm{~m}$ for microphone angle $\Theta=40^{\circ}$ (a) and $\Theta=60^{\circ}$ (b). Assessment of the symmetry pattern of the fan noise: positive angle (plain) and negative angle (dash). Fan noise (black) and background noise (gray).

aspect ratio $(L / c)$, the PSD of the sound pressure is derived as: ${ }^{39}$

$$
S_{p p}(\vec{x}, \omega)=\left(\frac{\omega c x_{3}}{2 \pi c_{0} S_{0}^{2}}\right)^{2} \frac{L}{2}\left|\mathcal{L}\left(\frac{\omega}{U_{c}}, \frac{\bar{k} x_{2}}{S_{0}}\right)\right|^{2} \Phi_{p p}(\omega) l_{y}(\omega) .
$$




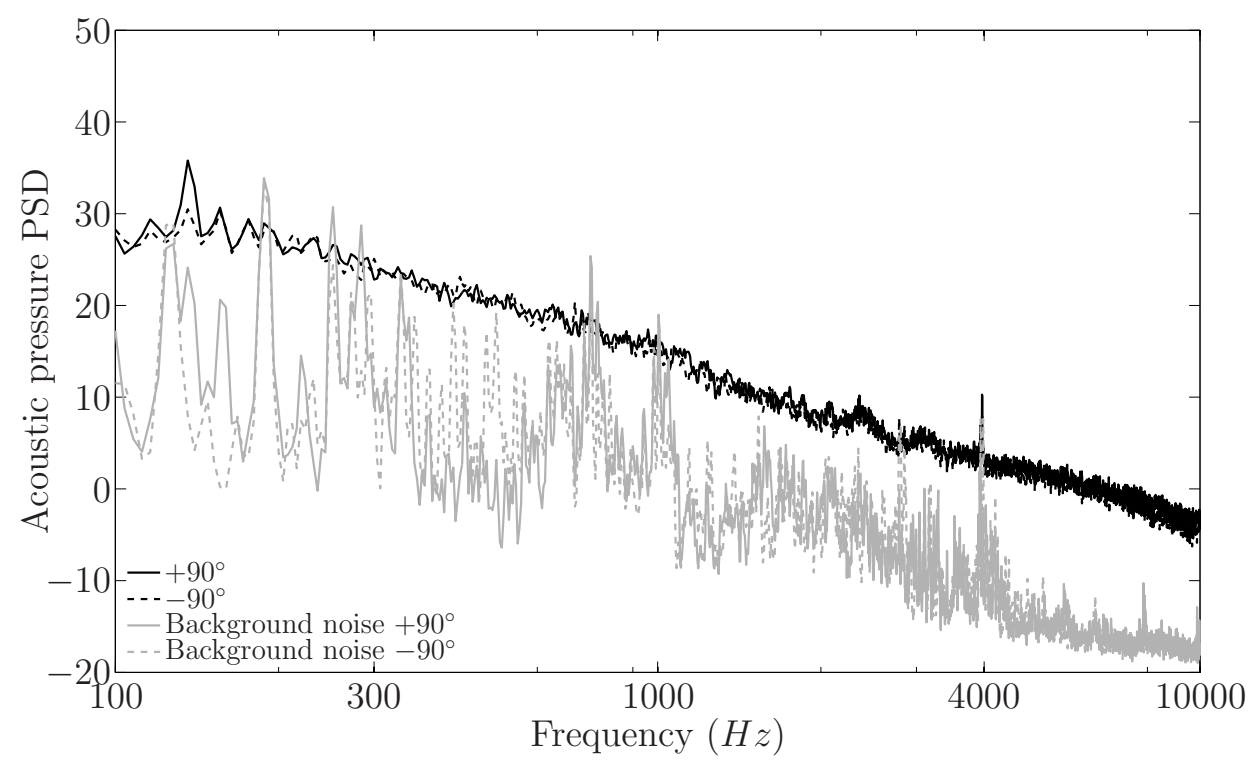

(c) $\Theta= \pm 90^{\circ}$

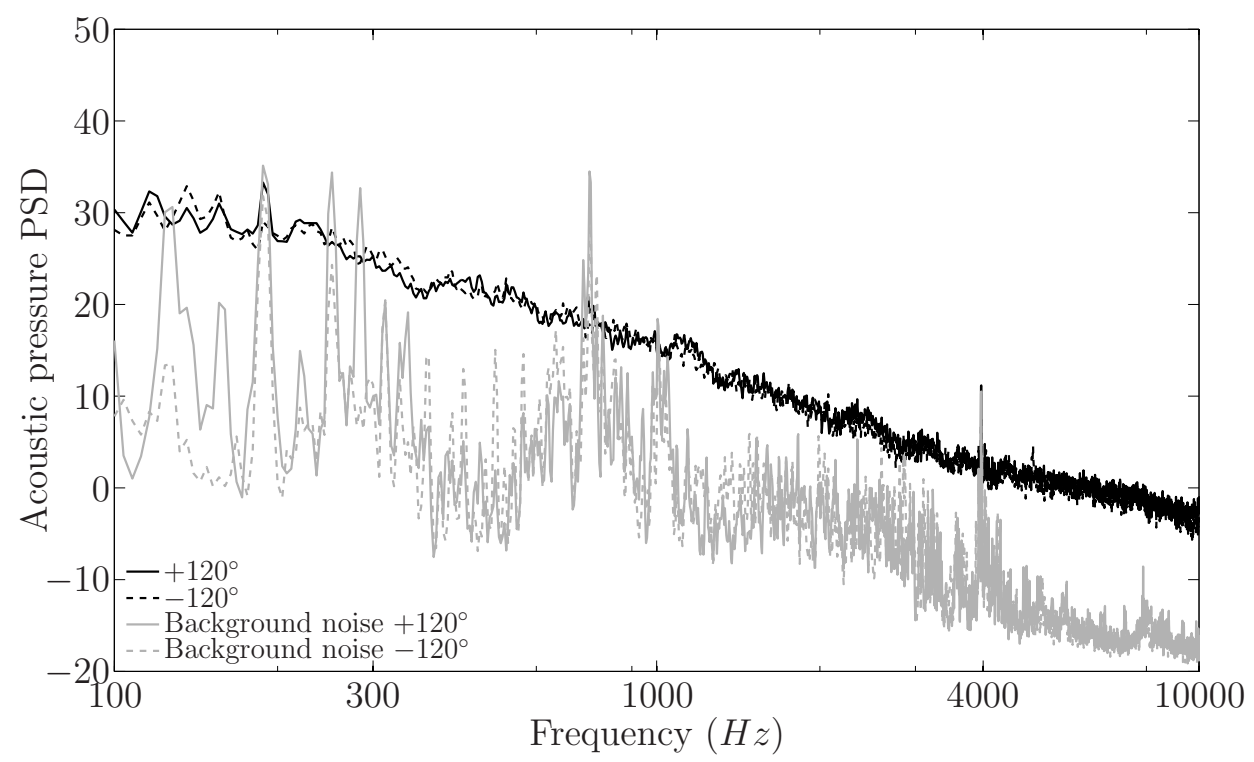

(d) $\Theta= \pm 120^{\circ}$

Figure 12: (Cont'd) Far field acoustic pressure PSD (dB/Hz ref. 2.10 ${ }^{-5} \mathrm{~Pa}$ ). Results at $R=1.7 \mathrm{~m}$ for microphone angle $\Theta=90^{\circ}$ (c) and $\Theta=120^{\circ}$ (d). Assessment of the symmetry pattern of the fan noise: positive angle (plain) and negative angle (dash). Fan noise (black) and background noise (gray).

Essentially it is determined by the wall-pressure statistics upstream of the trailing edge, including the wall-pressure spectrum $\Phi_{p p}$ and the corresponding spanwise correlation length 
$l_{y}$, and by the aeroacoustic transfer function $\mathcal{L}$ derived analytically. $\mathcal{L}=\mathcal{L}_{1}+\mathcal{L}_{2}$ where $\mathcal{L}_{1}$ is the main term as defined by Amiet $^{39}$ and $\mathcal{L}_{2}$ is the aforementioned back-scattering correction. ${ }^{38}$ The expressions of $\mathcal{L}_{1}$ and $\mathcal{L}_{2}$ are reproduced in appendix $\mathrm{A}$.

Eq. (5) is slightly different from the one derived by Amiet. Indeed an asymptotic analysis at low Mach number and at high frequency ${ }^{34}$ suggests that an extra factor 4 is needed to make Amiet's asymptotic solution coincide with Howe's model. ${ }^{2}$ The presence of this factor in eq. (5) has also been validated by comparison with experimental results on airfoils. A possible explanation based on the Kutta condition has been recently proposed by Moreau \& Roger. ${ }^{34}$

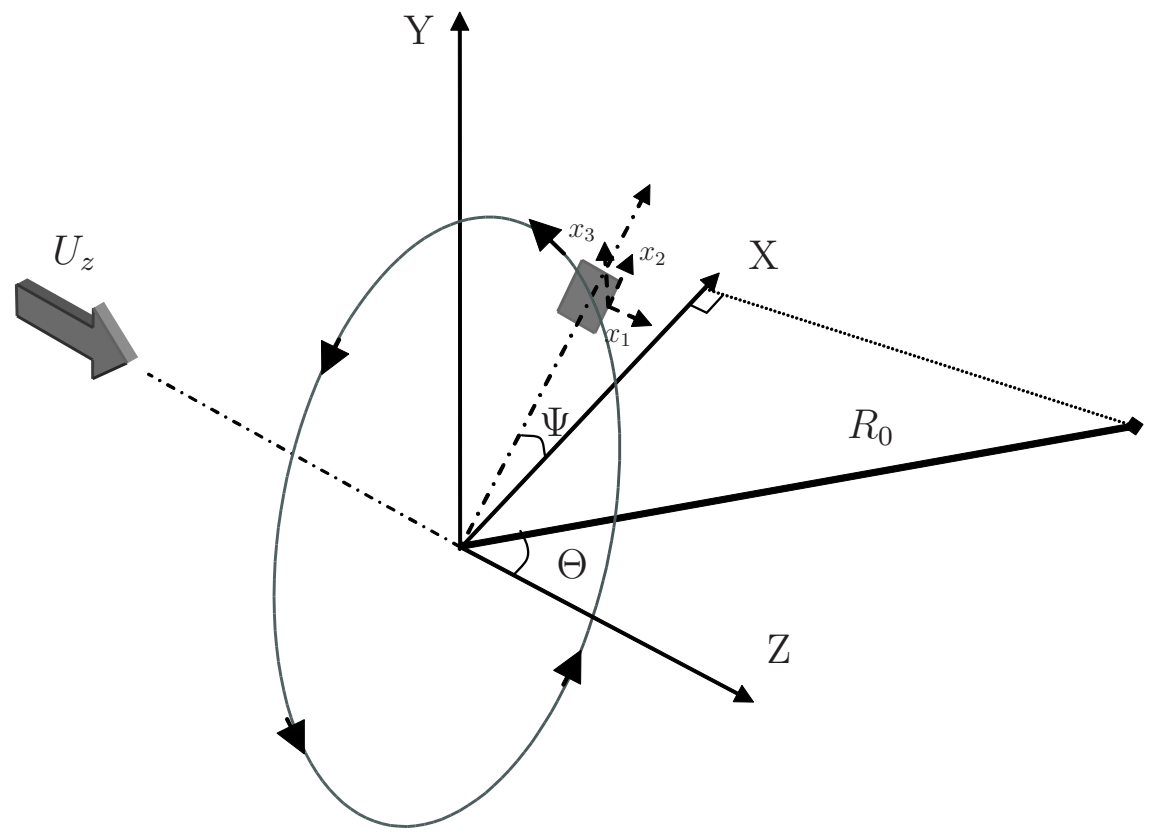

Figure 13: Coordinate system used in the rotating blade model.

Original Amiet's trailing-edge noise model for an isolated airfoil was extended by Schlinker $\&$ Amiet $^{10}$ to a low solidity helicopter rotor blade, based on a strip-theory approach. The same is made in the present study. Essentially the rotating blade is split into $n$ segments. The airfoil theory is applied to each segment, assimilating the circular motion to its locally tangent translating motion. This is only acceptable for sound frequencies much higher than the rotational frequency. ${ }^{1}$ More precisely, considering a blade segment according to the sketch of Fig. 13, the single-airfoil formulation (5) is first applied to calculate the radiated sound from one azimuthal location with no account of the relative motion with respect to the observer, but with the actual flow parameters relative to the segment according to the local velocity triangle. It must be noted that formulation (5) has been preferred to the more general one for arbitrary aspect ratio, involving a sine cardinal function. Indeed, it has been recently observed that using the general formulation, the noise predicted for a single airfoil can be slightly different from the cumulated noise as calculated by the sum of the 
contributions from a given number of sub-parts of the same airfoil. ${ }^{41}$ The relative motion of the blade is taken into account afterwards by adding a Doppler-factor correction expressed by Eq. (7) below. The mean velocity is assumed parallel to the chord line according to the weakly-loaded airfoil assumption of the linearized unsteady-aerodynamics theory. The observer can be placed in the $(X Z)$ plane (see Fig. 13) with no loss of generality. $\overrightarrow{R_{0}}$ is defined in the fixed reference frame by $\left(X_{o b s}=R_{0} \sin \Theta, Y_{o b s}=0, Z_{o b s}=R_{0} \cos \Theta\right)$. The first step consists in calculating observer's coordinates in the reference frame of the appropriate blade segment. The change of reference frame takes into account the angle of attack, the sweep angle $\Psi$ in the rotational plane and the stagger angle $\gamma_{s}=\pi / 2-\beta$. The stagger angle is defined with respect to the rotational plane, so that the blade is exactly in that plane when the stagger angle is zero. In the present application, the angle of attack is assumed zero in accordance with the unloaded-airfoil assumption and the blade is purely radial.

Observer's position in the moving reference frame is defined by the vector sum $\vec{x}=\overrightarrow{R_{0}}-$ $\overrightarrow{R_{A}}$ where $\overrightarrow{R_{A}}=(0 ; R ; 0)$ denotes the middle of the trailing-edge segment in the $\left(x_{1}, x_{2}, x_{3}\right)$ coordinate system (see Fig. 13). Eq. (5) gives the sound radiated by the segment, provided that observer's coordinates are expressed in the rotating reference frame and the frequency is corrected by the Doppler factor due to the relative motion. The instantaneous emitted frequency $\omega_{e}(\Psi)$ at the current position $\Psi=\Omega t$ is related to the received frequency $\omega$ by: ${ }^{42}$

$$
\frac{\omega}{\omega_{e}(\Psi)}=1+\frac{\overrightarrow{M_{t}} \cdot \widehat{O S}}{1-\overrightarrow{M_{r}} \cdot \widehat{O S}}
$$

where $M_{r}$ is the Mach number of the source relative to the fluid, $M_{t}$ is the Mach number of the source relative to the observer and $\widehat{O S}$ is the unit vector from the retarded source position to the observer. The present study applies to low-Mach number fans operating in a medium at rest. In this particular case, $U_{Z}=0$ and thus $M_{r}=M_{t}$, leading to the simplified expression:

$$
\frac{\omega_{e}(\Psi)}{\omega}=1+M_{t} \sin \Psi \sin \Theta
$$

in which $M_{t}=\Omega R / c_{0}$ is the rotational Mach number. The sound heard by the observer at a frequency $\omega$ is emitted at a frequency $\omega_{e}(\Psi)$ function of the angular position. The resulting spectrum must be calculated by averaging over all possible angular locations of the blade segment and by weighting with the Doppler ratio to be physically consistent with the necessary conservation of energy. This yields the following far-field noise PSD for a fan with B independent blades, assuming no blade-to-blade correlation for such a low-solidity fan: ${ }^{1}$

$$
S_{p p}(\vec{X}, \omega)=\frac{B}{2 \pi} \int_{0}^{2 \pi} \frac{\omega_{e}(\Psi)}{\omega} S_{p p}^{\Psi}\left(\vec{x}, \omega_{e}\right) d \Psi
$$


$S_{p p}^{\Psi}$ is the far-field pressure PSD due to a blade segment and is given by the single-airfoil theory (Eq. 5), where the observer coordinates are defined in the $\left(x_{1}, x_{2}, x_{3}\right)$ coordinate system. The integration over $\Psi$ is calculated by a recursive Newton-Cotes rule. In the following section, the model of reference ${ }^{10}$ with the back-scattering correction of reference ${ }^{38}$ is assessed by comparing with the experimental results.

\section{B. Validation}

Using the wall-pressure statistics measured in the aft part of the blades as input data according to the methodology discussed in section III, the analytical model is applied to the low-speed fan test case. The implementation of the model requires providing the blade geometry, the mean flow speed and the wall-pressure statistics for each blade segment, assuming local homogeneity. In the present application the blade is at least divided in three segments: one close to the hub, one around the mid-span and one close to the blade tip. The wall-pressure statistics for the hub and mid-span segments are both provided by the measurements made at mid-span, extrapolated on the basis of self-similar flows, whereas for the blade-tip segment they are directly provided by the blade-tip data. Then the extrapolation procedure is extended to a splitting into a larger number of segments. Firstly, the influence of the number of segments is reported in Fig. 14. A blade is divided in 3, 6, 9 and 12 segments. The spectrum obtained with 12 segments is chosen as a reference and the differences with this spectrum are plotted only for $\Theta=140^{\circ}$, other angles leading to the same conclusions. At each blade segment, the spectra are transposed to the local rotational speed, displacement thickness and wall shear stress using eq. (1). The convection speed is evaluated by the ratio $U_{c} / U$ from Table 2 and the local tangential speed $U=\Omega R$. The coherence length scale $l_{y}$ is deduced from a fitted with Corcos' model $\left(l_{y}(\omega)=b_{c} U_{c} / \omega\right)$. Since the blade geometry is almost constant at different radii, the far field spectra do not depend a lot on the number of segments. For $n=3$, the predicted sound level is roughly $0.5 \mathrm{~dB}$ lower than for the other three cases. Therefore, the blade will be divided only into 6 segments in the next, because a finer discretization would be time-consuming without being more accurate.

The variation of the acoustic spectrum with the rotational speed is now evaluated, rescaling the normalized wall-pressure spectra at various rotational speeds (cf. Fig. 15). The coherence length scale is obtained by Corcos'model, so that it decreases with increased velocity. For frequencies below $500 \mathrm{~Hz}$, this coherence length scale and consequently the trailing-edge noise prediction could be overestimated. The good agreement between the experimental results and the prediction could then be fortuitous and attributed to another unidentified mechanism. The good agreement at high frequencies show that the scaling laws used for the input data are valid over the whole speed range investigated here. 


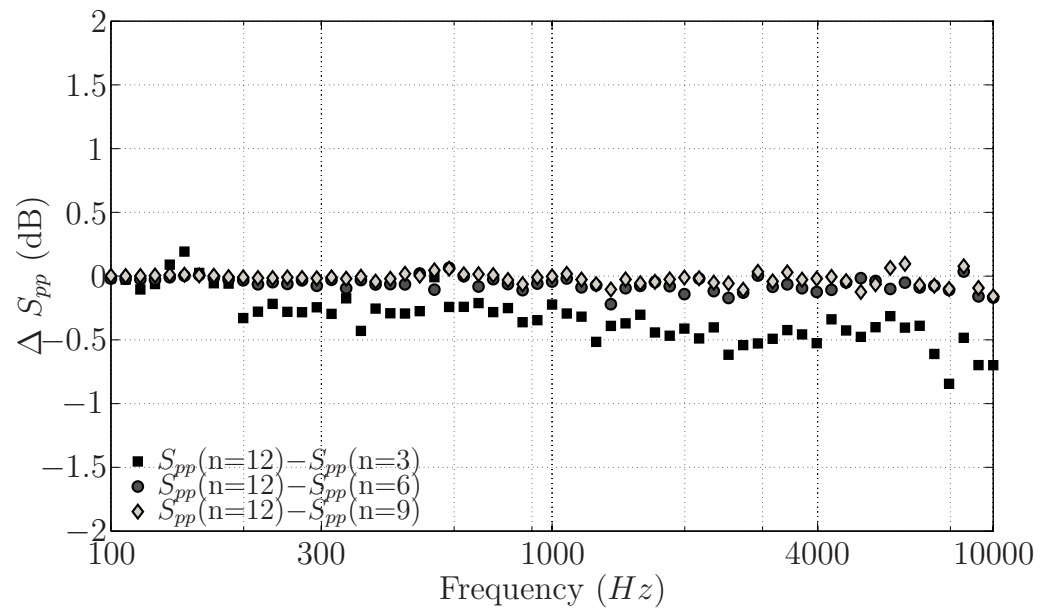

Figure 14: Influence of the blade segment number (n) on the prediction of acoustic pressure PSD at $\Theta=140^{\circ}$.

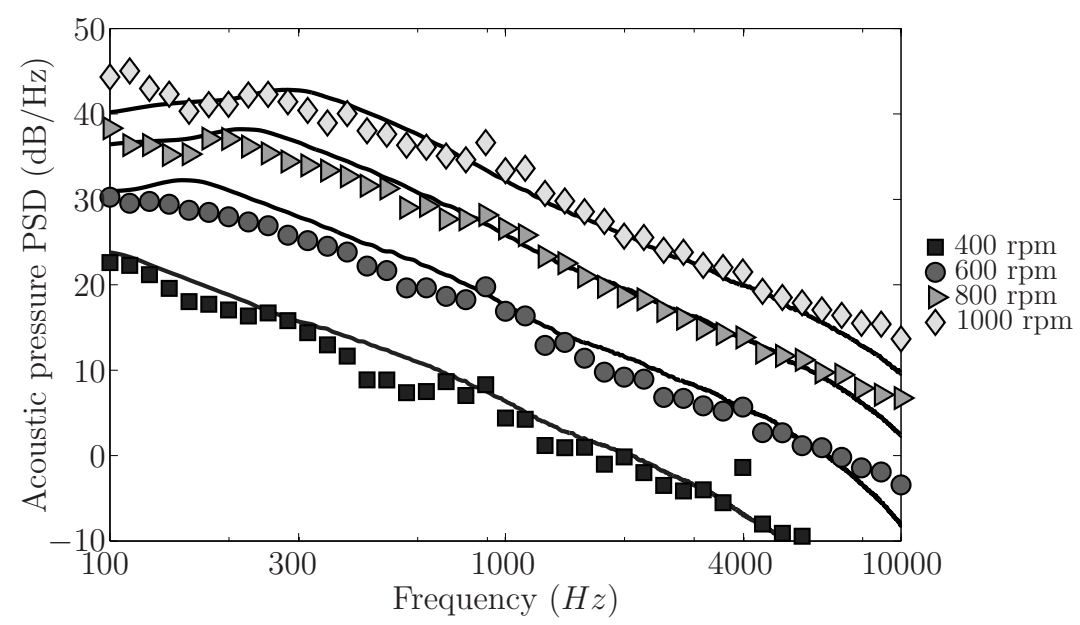

Figure 15: Acoustic pressure PSD in the rotational plane $\left(\Theta=90^{\circ}\right)$. Experimental results (symbols) and analytical results (lines). 
The analytical model is more widely compared with the experimental results on the radiation maps of Fig. 16, which show an overall good agreement. The cuts along the vertical dash-dotted lines produce the spectra at four angular positions plotted in Fig. 17 and the cuts along the horizontal dotted lines lead to the directivity pattern at four frequencies shown in Fig. 18.

In Fig. 17 the uncertainties have also been plotted. The errors are due to the experimental uncertainties and to the procedure to extrapolate the data on the 6 segments. Concerning the wall-pressure spectra, the experimental uncertainty is $0.2 \mathrm{~dB}$ as explained in section II.B. It is recalled that the procedure to rescale the wall-pressure spectra is based on a mean normalized wall-pressure from the mid-span set or the blade-tip set depending on the radial position. Based on the Fig. 5, it can be deduced that the error due to the rescaling is bigger in the low frequency range and can be evaluated to $\pm 1 \mathrm{~dB}$ maximum. The coherence length scale $l_{y}$ is also a source of error that has been evaluated to $30 \%$. Finally, the accuracy of the model is evaluated to $\pm 3 \mathrm{~dB}$ and the dashed lines in Fig. 17 correspond to this accuracy.

The prediction overestimates the noise radiated by the fan in the mid-frequency range as the observer position gets closer to the fan axis $\left(\Theta=40^{\circ}\right.$ and $\left.\Theta=120^{\circ}\right)$.



Figure 16: Angle-frequency directivity maps. Left: experiments. Right: analytical prediction. The vertical dash-dotted lines represent the spectra at 4 angular positions plotted in Fig. 17 and the horizontal dotted lines represent the directivity at 4 frequencies shown in Fig. 18. 


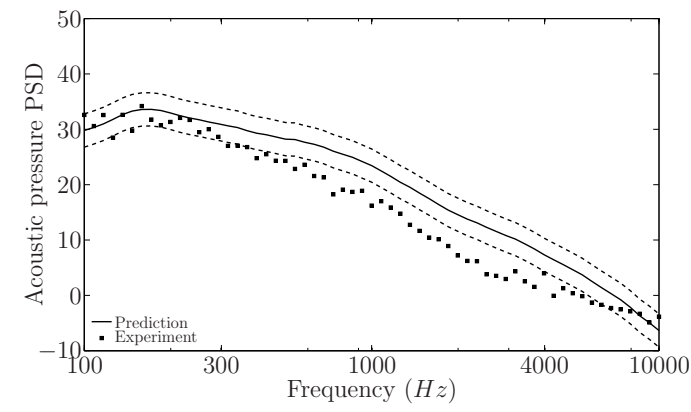

(a) $\Theta=40^{\circ}$

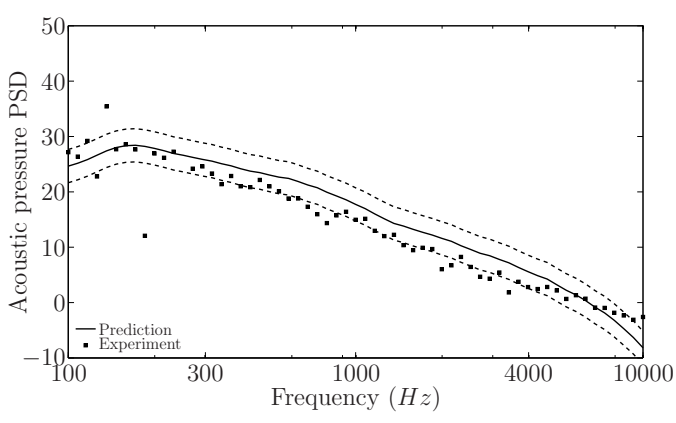

(c) $\Theta=90^{\circ}$

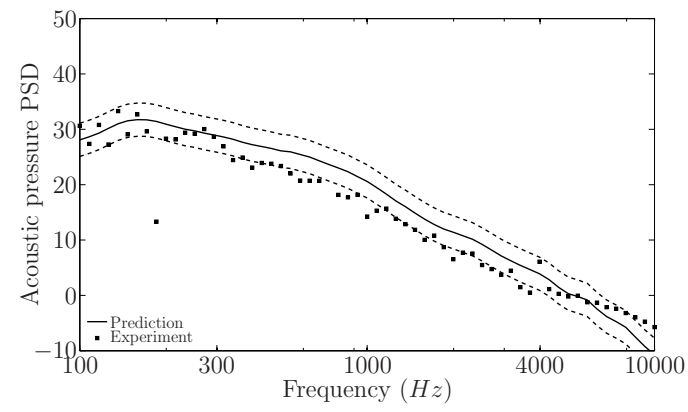

(b) $\Theta=60^{\circ}$

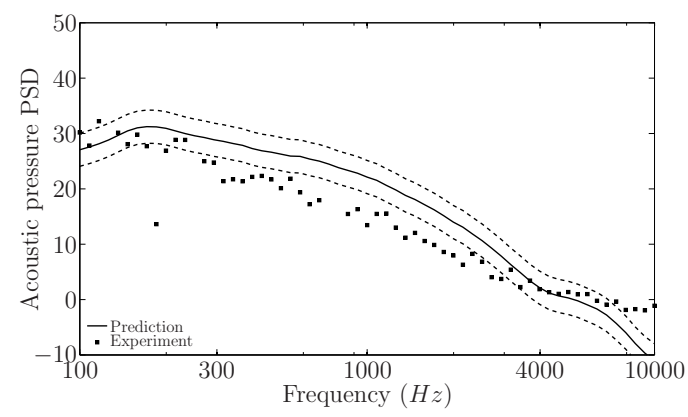

(d) $\Theta=120^{\circ}$

Figure 17: Far field acoustic pressure PSD (dB/Hz ref. $\left.2.10^{-5} \mathrm{~Pa}\right)$. Results at $R=1.7 \mathrm{~m}$ for four microphone angles $\Theta=\left[40^{\circ} ; 60^{\circ} ; 90^{\circ} ; 120^{\circ}\right]$. - Analytical prediction (with numerical accuracy - -). $\square$ Experimental results.

\section{Conclusion}

Analytical models aimed at predicting the trailing-edge noise radiated by stationary isolated airfoils have already been validated in the literature. ${ }^{21,35}$ In a previous attempt to extend the validation of one of them to a rotating blade, Schlinker \& Amiet $^{10}$ concluded that accurate predictions should refer to measured surface pressure data obtained near the trailing edge as input. In the continuation of the same methodology, the present dedicated experimental work resorts to a low-speed fan with instrumented blades to collect the wall-pressure statistics and the far-field acoustic pressure. A technology of embedded sensors has been developed and a particular calibration method has been found necessary to overcome the non-linearity of the sensors. The presently used analytical model is an extension of Schlinker \& Amiet's formulae including the back-scattering correction proposed by Roger \& Moreau. ${ }^{38}$ The data collected on the blades provide the necessary inputs. An overall agreement is obtained between the far-field measurements and the model predictions, with some overestimate as the observer gets closer to the fan axis. The effect of the number of segments has also been addressed, and in this particular case where the spanwise variation 


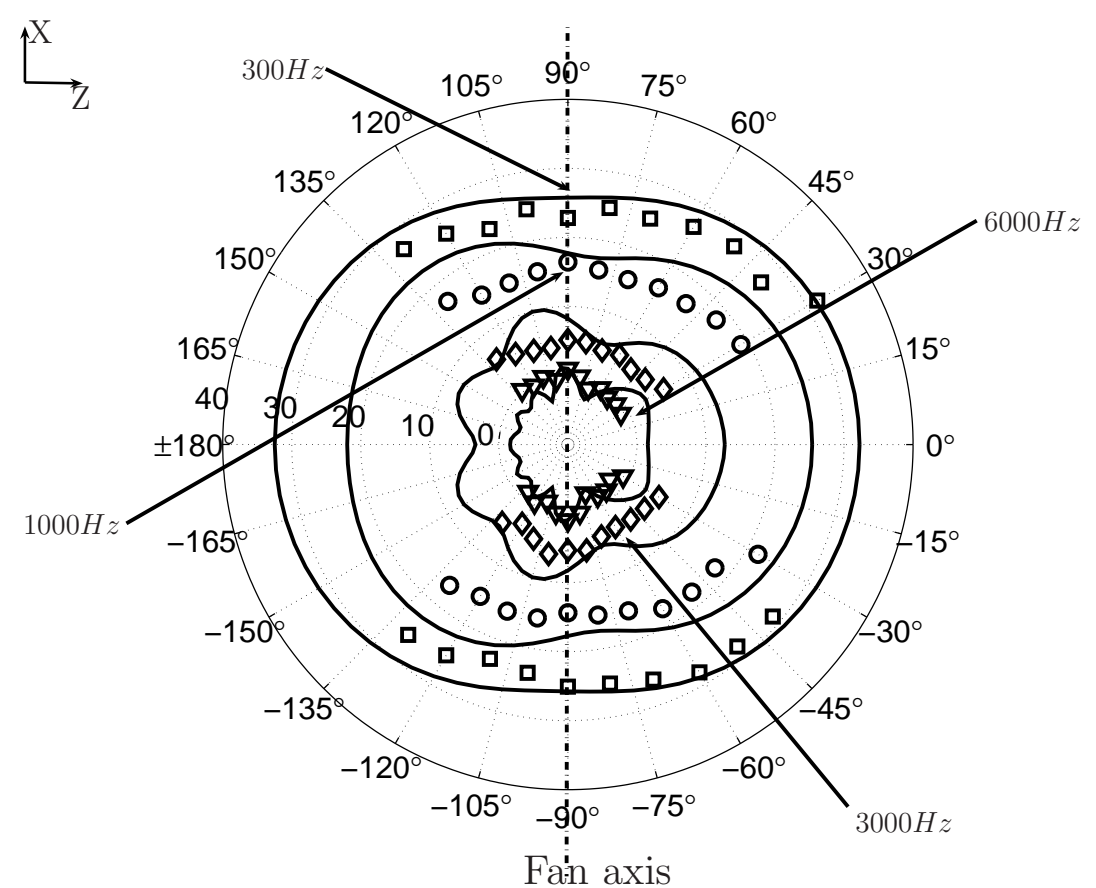

Figure 18: Fan directivity plot at 600 rpm for 4 frequencies: $f=[300 ; 1000 ; 3000 ; 6000] \mathrm{Hz}$. Experimental results (symbols) and analytical results (thick lines).

of the blade geometry is negligible (small twist and nearly constant chord), it does not affect the prediction of the far-field acoustic pressure. Finally, the present experiment provides a full database including wall-pressure statistics and far-field pressure to be compared to and/or used to validate later numerical simulation of the noise sources and of the acoustic field.

\section{Appendix A}

The extended solution presented by Roger \& Moreau ${ }^{38}$ has been implemented for this study. A main trailing edge contribution $\mathcal{L}_{1}$ and a first-order correction from the leading edge back-scattering $\mathcal{L}_{2}$ make up the total acoustic transfer function $\mathcal{L}$.

It leads to the following results :

$$
\mathcal{L}_{1}=-\frac{\mathrm{e}^{2 \mathrm{i} C}}{{ }_{\mathrm{i} C}}\left\{(1+\mathrm{i}) \mathrm{e}^{-2 \mathrm{i} C} \sqrt{\frac{B}{B-C}} E^{*}[2(B-C)]-(1+\mathrm{i}) E^{*}[2 B]+1\right\}
$$

with

$$
B=\bar{K}_{1}+\bar{\mu}(M+1) \quad, \quad C=\bar{K}_{1}-\bar{\mu}\left(x_{1} / S_{0}-M\right) \quad \text { and } \quad \bar{\mu}=\bar{K}_{1} M / \beta^{2}
$$




$$
\mathcal{L}_{2} \approx H\left\{\left[\mathrm{e}^{4 \mathrm{i} \bar{\mu}}\left(1-(1+\mathrm{i}) E^{*}[4 \bar{\mu}]\right)\right]^{c}-\mathrm{e}^{2 \mathrm{i} D}+\mathrm{i}[D+\bar{K}+\bar{\mu}(M-1)] G\right\}
$$

with

$$
\begin{aligned}
H & =\frac{(1+\mathrm{i}) \mathrm{e}^{-4 \mathrm{i} \bar{\mu}}\left(1-\Theta^{\prime 2}\right)}{2 \sqrt{\pi}(\alpha-1) \bar{K}_{1} \sqrt{B}}, \quad D=\bar{\mu}\left(1-x_{1} / S_{0}\right), \quad \epsilon=\left(1+\frac{1}{4 \bar{\mu}}\right)^{-1 / 2} \\
G & =(1+\epsilon) \mathrm{e}^{\mathrm{i}(2 \bar{\mu}+D)} \frac{\sin (D-2 \bar{\mu})}{D-2 \bar{\mu}}+(1-\epsilon) \mathrm{e}^{\mathrm{i}(-2 \bar{\mu}+D)} \frac{\sin (D+2 \bar{\mu})}{D+2 \bar{\mu}} \ldots \\
& +\frac{(1+\epsilon)(1-\mathrm{i})}{2(D-2 \bar{\mu})} \mathrm{e}^{4 \mathrm{i} \bar{\mu}} E^{*}[4 \bar{\mu}]-\frac{(1-\epsilon)(1+\mathrm{i})}{2(D+2 \bar{\mu})} \mathrm{e}^{-4 \mathrm{i} \bar{\mu}} E[4 \bar{\mu}] \ldots \\
& +\frac{\mathrm{e}^{2 \mathrm{i} D}}{2} \sqrt{\frac{2 \bar{\mu}}{D}} E^{*}[2 D]\left[\frac{(1-\epsilon)(1+\mathrm{i})}{D+2 \bar{\mu}}-\frac{(1+\epsilon)(1-\mathrm{i})}{D-2 \bar{\mu}}\right] \\
\Theta^{\prime} & =\sqrt{\frac{\bar{K}_{1}+\bar{\mu}(M+1)}{\bar{K}+\bar{\mu}(M+1)}}, \quad E^{*}[x]=\int_{0}^{x} \frac{\mathrm{e}^{-i t}}{\sqrt{2 \pi t}} \mathrm{~d} t, \quad[x+i y]^{c}=x+i \epsilon y
\end{aligned}
$$

\section{Acknowledgements}

The authors would like to thank Alain Guédel for his help during the experimental campaign. The present work was achieved as a collaboration between Valeo, CETIAT, SNECMA and Ecole Centrale de Lyon. The study was supported by the CIRT (Consortium Industrie-Recherche en Turbomachine), together with the French Ministry of Research.

\section{References}

${ }^{1}$ Paterson, R. W. and Amiet, R. K., "Noise of a Model Helicopter Rotor Due to Ingestion of Turbulence," Tech. Rep. CR - 3213, NASA, november 1979.

${ }^{2}$ Howe, M. S., "A review of the theory of trailing-edge noise," J. Sound Vib., Vol. 61, No. 3, 1978, pp. $437-465$.

${ }^{3}$ Brooks, T. F., Pope, D. S., and Marcolini, M. A., "Airfoil Self-Noise and Prediction," Tech. Rep. RP - 1218, NASA, 1989.

${ }^{4}$ Wang, M. and Moin, P., "Computation of Trailing-Edge Flow and Noise using Large-Eddy Simulation," AIAA Journal, Vol. 38, No. 12, 2000, pp. 2201-2209.

${ }^{5}$ Manoha, E., Troff, B., and Sagaut, P., "Trailing-Edge Noise Prediction using Large-Eddy Simulation and Acoustic Analogy," AIAA Journal, Vol. 38, No. 4, 2000, pp. 575-583.

${ }^{6}$ Casper, J. and Farassat, F., "Broadband trailing edge noise predictions in the time domain," J. Sound Vib., Vol. 271, 2004, pp. 159-176. 
${ }^{7}$ Zhou, Q. and Joseph, P. F., "A frequency domain numerical method for airfoil broadband self-noise prediction," J. Sound Vib., Vol. 299, 2007, pp. 504-519.

${ }^{8}$ Zhou, Q. and Joseph, P. F., "Frequency-Domain Method for Rotor Self-Noise Prediction," $A I A A$ Journal, Vol. 44, No. 6, 2006, pp. 1197-1206.

${ }^{9}$ Kim, Y. N. and George, A. R., "Trailing-Edge Noise from Hovering Rotors," AIAA Journal, Vol. 20, No. 9, 1982, pp. 1167-1174.

${ }^{10}$ Schlinker, R. H. and Amiet, R. K., "Helicopter Rotor Trailing Edge," Tech. Rep. CR - 3470, NASA, 1981.

${ }^{11}$ Moreau, S. and Roger, M., "Competing Broadband Noise Mechanisms in Low-Speed Axial Fans," AIAA Journal, Vol. 45, No. 1, 2007, pp. 48-57.

${ }^{12}$ Moreau, S. and Roger, M., "Effect of Airfoil Aerodynamic Loading on Trailing-Edge Noise Sources," AIAA Journal, Vol. 43, No. 1, 2005, pp. 41-52.

${ }^{13}$ Garcia Sagrado, A., Hynes, T., and Hodson, H., "Experimental Investigation Into Trailing Edge Noise Sources," Proceedings of the 12th AIAA/CEAS Aeroacoustics Conference, Cambridge, Massachusetts, No. AIAA-2006-2476, 2006.

${ }^{14}$ Rozenberg, Y., Roger, M., and Moreau, S., "Effect of Blade Design at Equal Loading on Broadband Noise," Proceedings of the 12th AIAA/CEAS Aeroacoustics Conference, Cambridge, Massachusetts, No. AIAA-2006-2563, 2006.

${ }^{15}$ Wang, M., Moreau, S., Iaccarino, G., and Roger, M., "LES prediction of pressure flcuctuations on a low speed airfoil," Center for Turbulence Research, Annual Research Briefs 2004, 2004.

${ }^{16}$ Moreau, S., Mendonca, F., Qazi, O., Prosser, R., and Laurence, D., "Influence of Turbulence Modeling on Airfoil Unsteady Simulations of Broadband Noise Sources," Proceedings of the 11th AIAA/CEAS Aeroacoustics Conference, Monterey, California, No. AIAA-2005-2916, 2005.

${ }^{17}$ Moreau, S., Neal, D., Khalighi, Y., Wang, M., and Iaccarino, G., "Validation of Unstructured-Mesh LES of the Trailing-Edge Flow and Noise of a Controlled-Diffusion Airfoil," Center for Turbulence Research, Proceedings of the Summer Program 2006, 2006.

${ }^{18}$ Rozenberg, Y., Roger, M., , Guédel, A., and Moreau, S., "Rotating Blade Self Noise: Experimental Validation of Analytical Models," Proceedings of the 13th AIAA/CEAS Aeroacoustics Conference, Roma, Italy, No. AIAA-2007-3709, 2007.

${ }^{19}$ Hutcheson, F. V. and Brooks, T. F., "Effects of Angle of Attack and Velocity on Trailing Edge Noise," Proceedings of the '42nd AIAA Aerospace Sciences Meeting and Exhibit' Conference, Reno, Nevada, No. AIAA-2004-1031, 2004.

${ }^{20}$ Ffowcs-Williams, J. E. and Hall, L. H., "Aerodynamic sound generation by turbulent flow in the vicinity of a scattering half-plane," J. Fluid Mech., Vol. 40, 1970, pp. 657-670.

${ }^{21}$ Brooks, T. F. and Hodgson, T. H., "Trailing Edge Noise Prediction from Measured Surface Pressures," J. Sound Vib., Vol. 78, No. 1, 1981, pp. 69-117.

${ }^{22}$ Corcos, G. M., "Resolution of Pressure in Turbulence," J. Acoust. Soc. Am., Vol. 35, No. 2, 1963 , pp. 192-199.

${ }^{23}$ Gilchrist, R. B. and Strawderman, W. A., "Experimental Hydrophone-Size Correction Factor for Boundary-Layer Pressure Fluctuations," The Journal of the Acoustical Society of America, Vol. 38, 1965, pp. 298-302. 
${ }^{24}$ Keith, W. L., Hurdis, D. A., and Abraham, B. M., "A Comparison of Turbulent Boundary Layer Wall-Pressure Spectra," ASME Journal of Fluids Engineering, Vol. 114, No. 3, 1992, pp. 338-347.

${ }^{25}$ Schlichting, H., Boundary-Layer Theory, McGraw-Hill, $6^{\text {th }}$ ed., 1968.

${ }^{26}$ Bradshaw, P., "'Inactive' motion and pressure fluctuations in turbulent boundary layers," J. Fluid Mech., Vol. 30, No. 2, 1958, pp. 241-258.

${ }^{27}$ McGrath, B. E. and Simpson, R. L., "Some Features of Surface Pressure Fluctuations in Turbulent Boundary Layers with Zero and Favorable Pressure Gradients," Tech. rep., NASA CR-4051, 1987.

${ }^{28}$ Monin, A. S. and Yaglom, A. M., Statistical Fluid Mechanics, Vol. 2, MIT Press, 1975.

${ }^{29}$ Blake, W. K., Mechanics of Flow-Induced Sound and Vibration, Applied Mathematics and Mechanics Volume 17-I and Volume 17-II, 1986.

${ }^{30}$ Gravante, S. P., Naguib, A. M., Wark, C. E., and Nagib, H. M., "Characterization of the Pressure Fluctuations Under a Fully Developed Turbulent Boundary Layer," AIAA Journal, Vol. 36, No. 10, 1998, pp. 1808-1816.

${ }^{31} \mathrm{Na}$, Y. and Moin, P., "The structure of wall-pressure fluctuations in turbulent boundary layers with adverse pressure gradient and separation," J. Fluid Mech., Vol. 377, 1998, pp. 347-373.

${ }^{32}$ Goody, M. and Simpson, R. L., "Surface Pressure Fluctuations Beneath Two- and Three-Dimensional Turbulent Boundary Layers," AIAA Journal, Vol. 38, No. 10, 2000, pp. 1822-1831.

${ }^{33}$ Corcos, G. M., "The structure of turbulent pressure field in boundary-layer flows," J. Fluid Mech., Vol. 18, 1964, pp. 353-378.

${ }^{34}$ Moreau, S. and Roger, M., "Back-scattering correction and further extensions of Amiet's trailing edge noise model. Part 2: Application," J. Sound Vib., Vol. 323, 2009, pp. 397-425.

${ }^{35}$ Roger, M. and Moreau, S., "Broadband Self-Noise from Loaded Fan Blades," AIAA Journal, Vol. 42, No. 3, 2004, pp. 536-544.

${ }^{36}$ Oerlemans, S. and Migliore, P., "Aeroacoustic Wind Tunnel Tests of Wind Turbine Airfoils," Proceedings of the 10th AIAA/CEAS Aeroacoustics Conference, Manchester, UK, No. AIAA-2004-3042, 2004.

${ }^{37}$ Stephens, D. B. and Morris, S. C., "The Effect of Blade Loading on Sound Sources in a Ducted Rotor," Proceedings of the 12th AIAA/CEAS Aeroacoustics Conference, Cambridge, Massachusetts, No. AIAA-2006-2683, 2006.

${ }^{38}$ Roger, M. and Moreau, S., "Back-scattering correction and further extensions of Amiet's trailing edge noise model. Part 1: Theory," J. Sound Vib., Vol. 286, No. 3, 2005, pp. 477-506.

${ }^{39}$ Amiet, R. K., "Noise due to turbulent flow past a trailing edge," J. Sound Vib., Vol. 47, No. 3, 1976, pp. 387-393.

${ }^{40}$ Amiet, R. K., "Acoustic radiation from an airfoil in a turbulent stream," J. Sound Vib., Vol. 41, No. 4, 1975, pp. 407-420.

${ }^{41}$ Christophe, J., Anthoine, J., Rambaud, P., and Moreau, S., "Numerical Issues in the Application of an Amiet Model for Spanwise-Varying Incoming Turbulence," Proceedings of the 14th AIAA/CEAS Aeroacoustics Conference, Vancouver, Canada, No. AIAA-2008-2865, 2008.

${ }^{42}$ Amiet, R. K., "Frame of Reference Considerations for the Forward Flight Noise Problem," Tech. Rep. N212775-1, UARL, December 1974. 